REVISTA MATEMATICA de la

Universidad Complutense de Madrid

Volumen L, números $1,2,3 ; 1988$

http://dx.doi.org/10.5209/rev_REMA.1988.v1.n1.18183

\title{
Geometric orbifolds
}

\author{
WILLIAM D. DUNBAR
}

\begin{abstract}
An orbifold is a topological space which «locally looks like» the orbit space of a properly discontinuous group action on a manifold. After a brief review of basic concepts, we consider the special case of 3-dimensional orbifolds of the form $\Gamma \mid M$, where $M$ is a simply-connected 3-dimensional homogeneous space corresponding to one of Thurston's eight geometries, and where $\Gamma<\operatorname{Isom}(M)$ acts properly discontinuously. A general description of these geometric orbifolds is given and the closed oriented geometric 3-orbifolds with $S^{3}$ as their underlying topological space are enumerated (except for hyperbolic orbifolds).
\end{abstract}

\section{INTRODUCTION}

A closed differentiable n-orbifold $\mathscr{O}^{n}$ is a Hausdorff topological space $X^{n}$ together with (compatible) modellings of neighborhoods of each point in $X^{n}$ on $\mathbb{R}^{n}$ /(finite subgroup of $\mathrm{O}(n)$ ), where the point corresponds to the equivalence class of the origin. The singular set of $\mathscr{O}^{n}$, denoted $\Sigma_{\vartheta^{n}}$, consists of points for which this finite group is not the trivial group. An oriented orbifold is one such that all the finite subgroups are contained in $S O(n)$ and such that the manifold-with-boundary $X^{n}-N\left(\Sigma_{0^{n}}\right)$ is oriented. Any closed 2-orbifold $\mathcal{O}^{2}$ can be specified by giving a compact surface $\left(=X^{2}\right)$, together with a finite number of distinguished points, cone points in the interior and corner points on the boundary, each labelled with an integer $>1$. The orbifold structure on $O^{2}$ is then given by charts $U \rightarrow \mathbb{R}^{2}$ (cyclic group of order $n$ ) at each cone point labelled "n" (where $U$ is a neighbourhood of the singular point), $U \rightarrow \mathbb{R}^{2} /($ dihedral group of order $2 n)$ at each corner point labelled «n», and $U \rightarrow \mathbb{R}^{2}$ /(reflection) at all other points on the boundary of the surface (so $\Sigma_{\mathscr{Q}^{2}}=\{$ cone points $\left.\} \cup \partial X^{2}\right)$. Any oriented closed 3-orbifold $\mathcal{O}^{3}$ can be specified by giving an oriented 3-manifold $\left(=X^{3}\right)$ containing a trivalent graph $\left(=\Sigma_{\sigma^{3}}\right)$, each edge of which is labelled with an integer $>1$ such that at each vertex, the labels $n_{1}, n_{2}, n_{3}$ associated to the three incident edges satisfy $1 / n_{1}+1 / n_{2}$

1980 Mathematics Subject Classification (1985 revision): 22E40.

Editorial de la Universidad Complutense. Madrid, 1988. 
$+1 / n_{3}>1$. This is because a finite subgroup of $S O(3)$ is either cyclic of order $n$ (corresponding to points on edges labelled «n»), dihedral of order $2 n$ (corresponding to a vertex with incident edges labelled $\langle 2\rangle,\langle 2\rangle,\langle(n))$, tetrahedral $(2,3,3)$, octahedral $(2,3,4)$, or icosahedral $(2,3,5)$.

An orbifold $\mathcal{O}^{n+m}$ fibers over a base orbifold $B^{n}$ with fiber orbifold $F^{m}$ if there is a map $\pi: \mathscr{O}^{n+n} \rightarrow B^{n}$ such that inverse images of neighborhoods in $B^{n}$ look like $\left(\mathbb{R}^{n} \times F^{m}\right) /($ finite group $G$ acting on both factors) and such that $\pi$ looks like projection on $\mathbb{R}^{n} / G$. In case $F^{m}=S^{1}$, we will call $\mathcal{O}^{n}$ a Seifert fibered orbifold $\left(\mathcal{O}^{3}\right.$ is a Seifert fibered orbifold with empty singular set iff it is a Seifert fibered manifold). Orbifolds are discussed in more detail in [Th2], [Sc], [BS2], [DM], and [Du].

There are eight 3-dimensional homogeneous spaces relevant to the study of compact 3-manifolds (and 3-orbifolds). Besides the 3-dimensional spherical, Euclidean, and hyperbolic geometries (denoted $S^{3}, E^{3}, H^{3}$ ), there are four geometries (denoted $S^{2} \times E^{1}, H^{2} \times E^{1}, T_{1}^{-}\left(H^{2}\right)$, and Nil) wich have natural foliations by straight lines (projecting along which yields $S^{2}, H^{2}, H^{2}, E^{2}$ respectively), and one geometry (denoted Solv) based on the only simplyconnected 3-dimensional Lie group wich is solvable, but not nilpotent. The explicit construction of the eight Lie groups that act transitively on these spaces is left as an exercise for the reader. The Lie groups should be "maximal» in some reasonable sense, e.g. we prefer to think of $S^{3}$ as $\mathrm{O}(4) / \mathrm{O}(3)$ (or $\mathrm{SO}(4) / \mathrm{SO}(3)$ if we restrict our attention to orientable 3 orbifolds) rather than as, say, $\mathrm{U}(2) / \mathrm{U}(1)$. A geometric orbifold is one that is diffeomorphic to $\Gamma \backslash(G / H)$, where $G / H$ is one of the above homogeneous spaces, and $\Gamma<G$ is a subgroup acting properly discontinuously on $G / H$ by left multiplication; equivalently, an orbifold is geometric if it admits an atlas of charts (from local universal covers) into the geometry such that the transition maps (and local group actions) are restrictions of isometries. The eight geometries are discussed in more detail in [Sc].

Although orbifolds are locally the orbit spaces of finite group actions on manifolds, an orbifold is not necessarily globally the orbit space of a finite group action (or even a properly discontinuous group action) on some manifold. An orbifold is bad if its universal cover has non-empty singular set (otherwise it is good). All 1-orbifolds are good, and the bad closed 2-orbifolds are classified in [Th2, Chapter 13]. It is a consequence of a geometric decomposition for irreducible 3-orbifolds with singular set of dimension $\geqslant 1$ [Th4] that irreducible 3-orbifolds which have no bad 2-suborbifolds are good (cf. [MM]); in the case of 3-orbifolds having singular set consisting of isolated points which are modelled on $\mathbb{R}^{3}$ /(antipodal map), which are the ones left out of the above theorem, the orientable double cover has empty singular set.

The author would like to acknowledge the helpful remarks of the referee. This paper contains the results of the author's Ph. D. dissertation (and extends them to other geometries). 


\section{MOTIVATION}

Orbifolds provide a convenient way of "encoding" properly discontinuous group actions on manifolds which are not necessarily free. The natural notion of covering space for orbifolds is such that, for example, the projection from any manifold to its quotient by a properly discontinuous group action can be thought of as a covering map. The "complexity" of an orbifold is a combination of the complexity of its singular set and the complexity of its underlying topological space. Roughly, the former reflects the extent to which the fundamental group of the orbifold (defined as the group of covering transformations of its universal cover) is generated by elements with fixed points, and the latter reffects the "rest» of the group. If $N$ is the normal closure in $\pi_{1}^{\text {orb } b}\left(\mathcal{O}^{n}\right)$ of the covering transformations acting with fixed points, then $\pi_{1}\left(X^{n}\right) \simeq \pi_{1}^{\text {arb }}\left(\mathcal{O}^{n}\right) / N$. Consequently, closed 3-orbifolds whose underlying topological spaces are simply-connected correspond to group actions generated by elements with fixed points. If the orbifold is orientable and admits a geometric structure modelled on a geometry other than $\mathrm{H}^{3}$, one can show (following [Se] for the most part) that $X^{3}$ must be homeomorphic to $\mathrm{S}^{3}$. On the other hand, the analogous statement for hyperbolic orbifolds is equivalent to the Poincare Conjecture, since one can use the existence of universal links which are hyperbolic [HLM] to show that any closed orientable 3-manifold is the underlying space of a hyperbolic orbifold.

There are other reasons to consider such orbifolds: they provide an endless variety of examples that can easily be twisted and manipulated, where "twistedness" of the singular set (now a labelled trivalent graph in $\mathrm{S}^{3}$ ) in some sense plays the role of the topology of a 3-manifold. In the special case where the singular set is a knot labelled with the integer $n$, finding a geometric structure for this orbifold immediately implies that the $n$-fold cyclic branched cover of the knot has a structure modelled on the same geometry (in which the action of $\mathbb{Z}_{n}$ upstairs can be taken to be by isometries).

Geometric orbifolds are conjectured to be the basic building blocks out of which a general compact 3-orbifold may be constructed, in the same sense that a general compact 3-manifold is conjectured to be constructable (via connected sums and gluing boundary tori together) out of geometric 3manifolds. These conjectures have been shown to be true under fairly general circumstances (Haken manifolds [Th3], orbifolds with singular set of dimension $\geqslant 1[\mathrm{Th} 4]$ ). One purpose of this article is to give a better idea of what the "trivial" ( = non-hyperbolic) pieces of the geometric decomposition of a 3-orbifold look like. Also, since a 3-orbifold with $X^{3} \simeq S^{3}$ can often be shown by combinatorial methods to be geometrically indecomposible, the tables at the end of this article can be used to give and indirect proof that a given such orbifold is hyperbolic (by showing that it is geometric, e.g. via [Th4], and doesn't appear in the tables). 
Henceforth, unless otherwise specified, ALL 3-ORBIFOLDS will be assumed to be COMPACT, CONNECTED, ORIENTED, and WITHOUT BOUNDARY; all 1- and 2-orbifolds wil be assumed to be compact, connected, and without boundary (but note that $\partial X^{n}$ can be non-empty when $\mathcal{O}^{n}$ is closed and non-orientable).

\section{ROUGH CLASSIFICATION OF GEOMETRIC ORBIFOLDS}

Theorem 1. Suppose $\mathcal{O}^{3}$ is a geometric orbifold. Then exactly one of the following holds:

1) $\mathscr{O}^{3}$ is a hyperbolic orbifold.

2) $\mathcal{O}^{3}$ is a Seifert fibered orbifold with base $\mathcal{O}^{2}$, in which case one of the following descriptions applies (both may apply if $\mathcal{O}^{3}$ fibers in more than one way):

a) if $\partial X^{2}=\varnothing\left(X^{2}\right.$ denotes the underlying space of $\left.\mathcal{O}^{2}\right)$, then $X^{3}$ is a closed Seifert fibered manifold, and $\sum_{0^{3}}$ consists of fibers (labelled with various integers $>1$ );

b) if $\partial X^{2}$ has $n$ components ( $n>0$ ), then $X^{3}$ is the result of gluing solid tori to a Seifert fibered manifold with $n$ boundary tori, such that the meridians of the tori are glued to fibers on the boundary. $\sum^{\prime}$ consists of fibers, plus a Montesinos link (labelled «2», and slightly modified) inside each added solid torus (more on this in \$4).

3) $\mathcal{O}^{3}$ is a solvorbifold; in particular, it fibers over either $S^{1}$ or $S^{1} / r e f l e c t i o n$, with fiber either $T^{2}$ or $T^{2} /\left(180^{\circ}\right.$ rotation fixing 4 points $)=$ $\left(S^{1} \times S^{1}\right) /($ reflection of both factors).

4) $\mathcal{O}^{3}$ is one of the 12 Euclidean orbifolds $\left(11\right.$ with $X^{3} \simeq S^{3}$ and 1 with $X^{3} \simeq \mathbb{R} P^{3}$ ) or 18 spherical orbifolds (all with $X^{3} \simeq S^{3}$ ) which do not fiber over 2-orbifolds.

Furthermore, no 3-orbifold possesses structures from two different geometries.

Proof: The argument in [Sc, Thm 5.2] (for manifolds) serves to iustify the last setence of the theorem. All 3-orbifolds modelled on $H^{2} \times E^{1}, T_{1}\left(H^{2}\right)$, and Nil are of type 2 as a consequence of the fact the isometry groups of these geometries preserve fibrations by lines (which become fibrations by circles generically - and intervals in any orientable compact quotient space, cf. [Sc, Thm 4.13, Thm 4.15, Thm 4.16]). Most Euclidean and spherical 3-orbifolds are of type 2 for a similar reason; there is no fibration preserved by the entire isometry group, but most discrete subgroups will preserve some family of parallel lines in $E^{3}$, or some Hopf fibration of $S^{3}$. All 3-orbifolds modelled on $S^{2} \times E^{1}$ can be remodelled if necessary so that the fibration by lines ${ }^{*} \times E^{1}$ 
induces a Seifert fibering of the orbifold, so such an orbifold is of type 2 as well; this can be proven by brute force analysis of the discrete subgroups of the groups of isometries of $S^{2} \times E^{1}$. Similar analysis of the isometries of Solv justifies the statements made about type 3 (cf. [Sc, Thm 4.17]). The numbers in the description of type 4 are based on the algebraic classifications of Euclidean and spherical crystallographic group; see $\S 7$ and $\S 8$ for details.

Remark. Type 4 does not arise in the consideration of geometric manifolds, since it turns out that all Euclidean and spherical 3-manifolds can be Seifert-fibered;

Like oriented circle bundles over surfaces, an oriented Seifert fibered 3orbifold has an Euler number $\mathrm{e}$; $\mathrm{e}$ is a rational number which describes an obstruction to finding a section, and like the usual Euler number, multiplies if you pull back along a covering of the base and divides if you unwrap the fiber; see [Sc, §3], [BS3] or [Du] for details. Given a particular Seifert fibered orbifold (type 2), the table in Figure 1 shows which geometry it should fall under, merely in terms of the Euler characteristic $\chi$ of the base 2-orbifold and the Euler number e of the bundle.

\begin{tabular}{c|c|c|c} 
& $\chi>0$ & $\chi=0$ & $\chi<0$ \\
\hline$e=0$ & $S^{2} \times E^{1}$ & $E^{3}$ & $H^{2} \times E^{1}$ \\
\hline$e \neq 0$ & $S^{3}$ & Nil & $\left.T_{1} \tilde{(} H^{2}\right)$
\end{tabular}

The construction of a geometric structure of the "right" type on a Seifert fibered manifold (equivalent to finding a discrete faithful representation of the fundamental group into the isometry group of the geometry) is done in [Sc, Thm 5.3]. The same techniques work in the orbifold setting, with the exception that Seifert fibered orbifolds with a bad base 2-orbifold and with Euler number $=0$ are also bad, hence do not admit the $S^{2} \times E^{1}$-structure predicted by the table. On the other hand, if the Euler number is non-zero, the orbifold does have the predicted spherical structure.

Proposition 2. Suppose that $\mathcal{O}^{3}$ Seifert fibers over a bad 2-orbifold $\mathcal{O}^{2}$ with non-zero Euler number $e$; then

1) $\pi_{1}^{\text {orb }}\left(\mathcal{O}^{3}\right)$ is finite.

2) $\mathcal{O}^{3}$ is good.

3) $\mathcal{O}^{3}$ admits another Seifert fibering over a spherical 2-orbifold which is either a sphere with 2 cone points labelled $\langle n\rangle, n \geqslant 1$, or a disk with 2 corner points labelled $\langle n\rangle, n \geqslant 1$.

4) $0^{3}$ admits a spherical structure. 
Proof. To prove part (1), it suffices to consider the case where $\mathcal{O}^{2}$ is a sphere with a single cone point, or with two cone points labelled with different integers. $X^{3}$ is then a lens space $\left(\neq S^{2} \times S^{1}\right.$ since $\left.e \neq 0\right)$, and $\Sigma_{\mathcal{E}^{3}}$ is contained in the cores of the two solid tori into which $X^{3}$ can be decomposed. Hence $\mathcal{O}^{3}$ is finitely covered by an orbifold $\widetilde{O}^{3}$ with underlying space $S^{3}$, having singular set either empty, an unknotted circle, or a 2-component Hopf link, which in turn is finitely covered by $S^{3}$. This shows part (1), and the remaining parts follow from [DM, Remark 3.1 and Theorem 5.1].

Similarly, given a description of a 3-orbifold as a bundle over $S^{1}$ with fiber orbifold $F$ and monodromy $\varphi: F \rightarrow F$, the table in Figure 2 shows which geometry it should fall under, in terms of the Euler characteristic $\chi$ of $F$ and the class $[\varphi]$ represented by $\varphi$ in the mapping class group of $F$.

\begin{tabular}{l|c|c|c}
\hline$[\varphi]$ finite order & $\chi>0$ & $\chi=0$ & $\chi<0$ \\
\hline$[\varphi]$ reducible & $S^{2} \times E^{1}$ & $E^{3}$ & $H^{2} \times E^{1}$ \\
\hline$[\varphi]$ (pseudo-) Anosov & & Nil & hybrid \\
\hline Solv & $H^{3}$
\end{tabular}

FIGURE 2

The term "hybrid" refers to the fact that in general, such a bundle will have a geometric decomposition into a number of hyperbolic and $H^{2} \times E^{1}$-manifolds with boundaries consisting of tori (or finite quotients of tori) with the various boundary components identified in pairs. Of course, the lower-right-most entry is a much deeper fact than the others; see [Th3]. The second column is the one most relevant to the present article, since it explains why there are exceptional braids in the descriptions of solvorbifolds, and suggests (correctly) that the exceptions are listed elsewhere, under other geometries. [Sc, Thm $5.3]$ is also relevant here, and there is a similar caveat concerning bad 2orbifolds: if the fiber is bad, the bundle will be bad, and the predicted $S^{2} \times E^{1}$ structure will not exist.

\section{RATIONAL TANGLES AND MONTESINOS GRAPHS}

We need to develop a notation for describing «small» pieces of the singular set of a 3-orbifold of type $2 b$ in Theorem 1 of the previous section, for use in the tables that follow. These pieces arise as the intersection of the singular set with 3-balls whose boundaries hit the singular set in four points. 
If $m$ and $n$ are positive integers such that $0<m \leqslant n / 2$, there is exactly one way to write $m / n$ as a continued fraction

$$
m / n=\frac{1}{k_{1}+\frac{1}{k_{2}+\ddots}+\frac{1}{k_{l}}}
$$

if we take $k_{1}, \ldots, k_{l}$ to be positive integers, and require that $k_{l}$ be $\geqslant 2$. We define the tangled graph with parameter $m, n$ as in Figure 3 below. When $m$ and $n$ are relatively prime, this is just a rational tangle; in Conway's notation [Co], it would be denoted by $k_{l} k_{l-1} \ldots k_{2} k_{1} 0$.
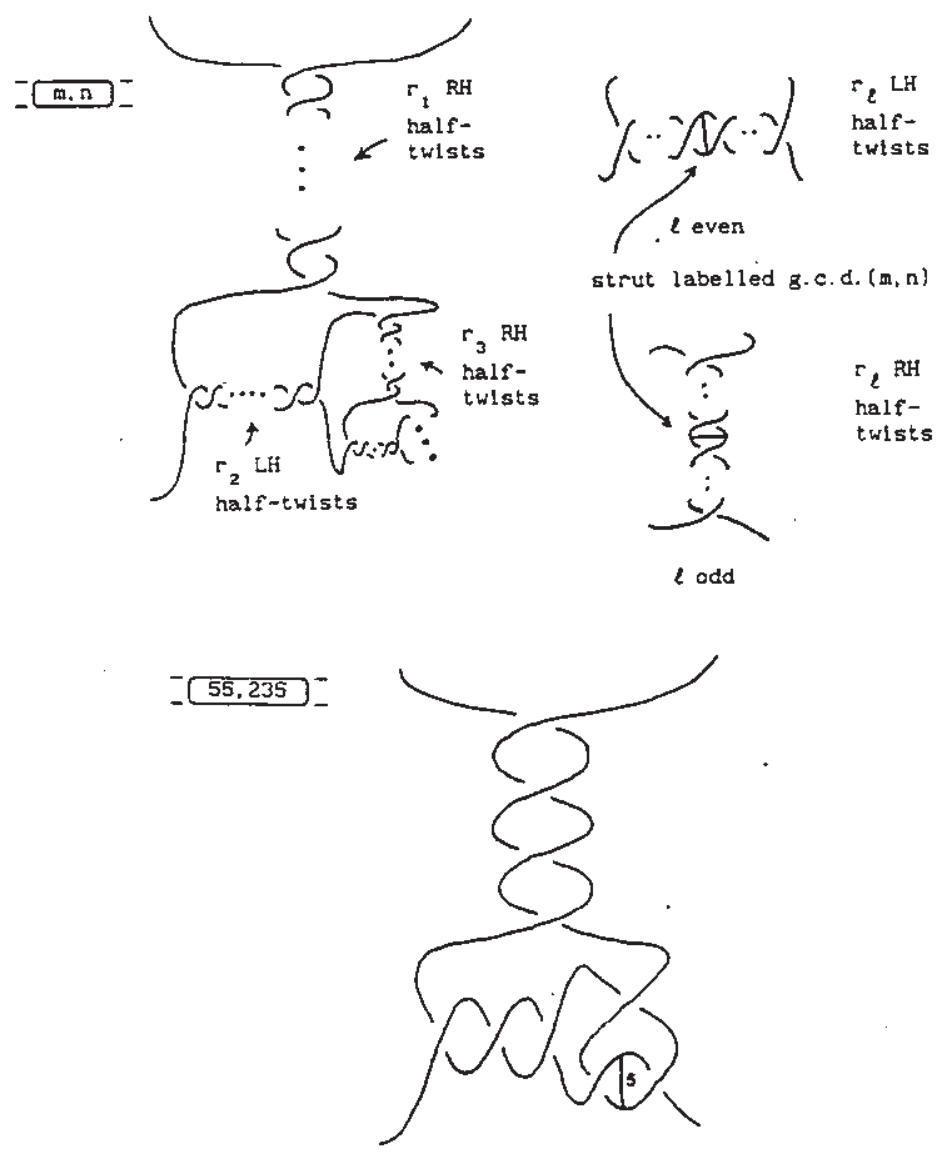

FIGURE 3 
As an aid to sketching, note that all twists have «positive slope», in the sense that all over-crossings are from lower left to upper right.

This tangle should be thought of as the singular set of a 3-orbifold-withboundary, with underlying space a 3-ball, which Seifert fibers over one of the 2-orbifolds-with-boundary $D^{2} /$ (dihedral group) (i.e., over a regular neighborhood of a corner point). The edges of the singular set upstairs are all labelled "2"), except for the strut in the innermost twist, which is labelled g.c.d.(m,n) (or omitted, if $m$ and $n$ are relatively prime). The strut is an exceptional fiber sitting over the corner point; a typical fiber over a boundary point is a circle on the boundary of the 3-ball isotopic to the great circle in the $y z$-plane (if the plane of the paper in Figure 3 is taken to be the $x y$-plane). This fibration corresponds to an action of the dihedral group of order $2 n$ $\left(=<\sigma, \tau: \sigma^{2}=\tau^{2}=(\sigma \tau)^{n}=1>\right)$ on $D^{2} \times S^{1}$, where $\sigma$ and $\tau$ act on $D^{2}$ as reflections in lines making an angle of $+\pi / n$ (measured from $\sigma$ to $\tau$ ), and where $\sigma$ and $\tau$ act on $S^{1}$ as reflections in lines making an angle of $-m \pi / n$ (measured from $\sigma$ to $\tau$ ). The sign of $m / n$ is well-defined, since once an orientation is fixed on $D^{2} \times S^{1}$, orientations on the factors are determined, up to the operation of reversing both.

We can extend this notation to the case of $-n / 2 \leqslant m<0$ by reversing all crossings in the tangle with parameter $-m, n$. It is also convenient to define a tangle with parameter $0, n$ (for $n>1$ !) to be two horizontal arcs (labelled « 2 ) with a vertical strut labelled «n»».

Finally, an integer $k$ enclosed in a box will denote two arcs labelled «2», with $k$ LEFT-handed half-twists along a horizontal axis (if $k<0$, use $|k|$ righthanded half-twists).

As before, twists with positive parameters have positive slopes.

Joining a bunch of these tangles together in a circle, one obtains a 3 orbifold-with-boundary whose underlying topological space is a solid torus, and which fibers over a 2-orbifold-with-boundary as shown below in Figure 4.

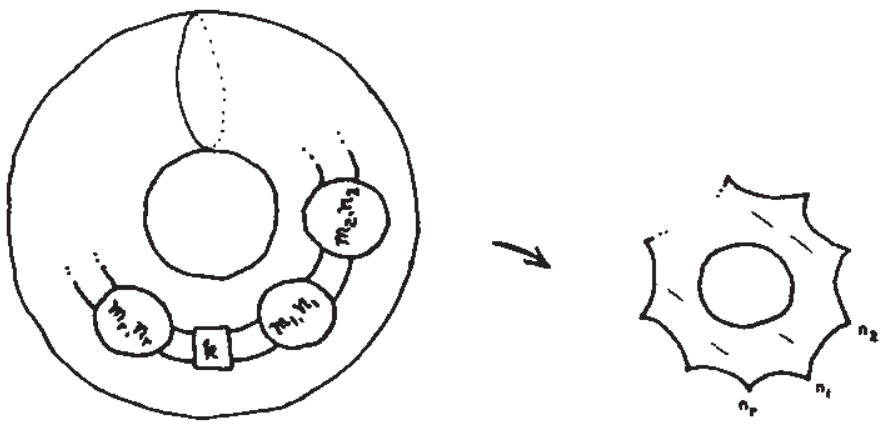

FIGURE 4 
The fibers on the boundary of the solid torus are meridians. The singular set is a Montesinos link (cf. [Mo], [Oe], [BZ]) if g.c.d. $\left(\mathrm{m}_{i}, \mathrm{n}_{i}\right)=1$ for $1 \leqslant i \leqslant r$, and otherwise is a trivalent graph which we can refer to as a Montesinos graph. These are the "plugs" which are glued to Seifert fibered manifolds with boundary to form geometric orbifolds of type $2 \mathrm{~b}$ in $\$ 3$.

\section{TYPE 2 ORBIFOLDS WITH UNDERLYING SPACE $\mathrm{S}^{3}$}

If the underlying topological space of. a Seifert fibered orbifold $\mathcal{O}^{3}$ is simply-connected, it is not hard to see that the underlying space of $\mathcal{O}^{3}$ is $S^{3}$ and that the 2 -orbifold which $\mathcal{O}^{3}$ projects to must be either topologically a $2-$ sphere, with a certain number of cone points, or topologically a 2-disk, with a certain number of cone and corner points (half of the proof is in [Se] and the rest in [Du, Proposition 2.14]). We can denote the former by $S^{2}(\ldots$ unordered labels of cone points...) and the latter by $D^{2}(\ldots$ unordered labels of cone points...; ...cyclically ordered labels of corner points...). In the former case, the singular set consists of fibers in some Seifert fibration of $S^{3}$, i.e. a collection of "parallel» torus knots, plus possibly one or both of the «axes»; links of this type are discussed in [BM]. It is easy to check that the Euler numbers of all such Seifert fibered orbifolds must be nonzero (essentially it is a consequence of the fact that the left and right Hopf fibrations of $S^{3}$ over $S^{2}$ have Euler numbers +1 and -1 respectively). Using the chart in Figure 1, we see that the appropriate geometry is $S^{3}$, Nil or $T_{1}^{-}\left(H^{2}\right)$, depending on whether the Euler characteristic of the base orbifold is positive, zero or negative. This Euler characteristic is easy to compute from a picture of the 3-orbifold, since the Seifert fibering of $S^{3}$ by $(p, q)$ torus knots corresponds to a projection to the 2-orbifold $S^{2}(|p|,|q|)$. Adding a torus knot labelled «k» to the singular set introduces a new cone point labelled « $k$ » downstairs; adding an axis labelled $\langle k »$ to the singular set changes the label on one of the original cone points from $|p|$ to $|p| k$ or from $|q|$ to $|q| k$, as appropriate.

In the latter case, we can construct a model for the 3-orbifold by building on our description of "plugs" for geometric orbifolds of type $2 b$ in the last section. By gluing $D^{2} \times S^{1}$ to the solid torus in Figure 5 (identifying the boundaries, meridian to longitude), we get a 3 -orbifold with underlying space $S^{3}$ and singular set a Montesinos graph, fibering over a 2-orbifold with underlying space $D^{2}$ and singular set $=\partial D^{2}$, with $r$ corner points modelled on $\mathbb{R}^{2} /\left(\right.$ dihedral group of order $\left.2 n_{i}\right), 1 \leqslant i \leqslant r$. The Euler characteristic of this 2 orbifold is easily calculated to be $1+(1 / 2) \sum_{i=1}^{r}\left(-1+1 / n_{i}\right)$ (e.g., via cut-andpaste arguments in the orientable double cover). The notation has been contrived so that this orbifold has Euler number $e=(-1 / 2)\left(k+\sum_{i=1}^{r} m_{i} / n_{i}\right)$; cf. [BS3], which denotes the Euler number by $e_{0}$. As a final embellishment, one can incorporate a finite number of the generic fibers into the singular set, labelling them with integers $d_{1}, d_{2}, \ldots, d_{s}>1$. The base 2 -orbifold will now have $s$ cone points, locally modelled on $\mathbb{R}^{2}$ /(cyclic groups of rotations of order 

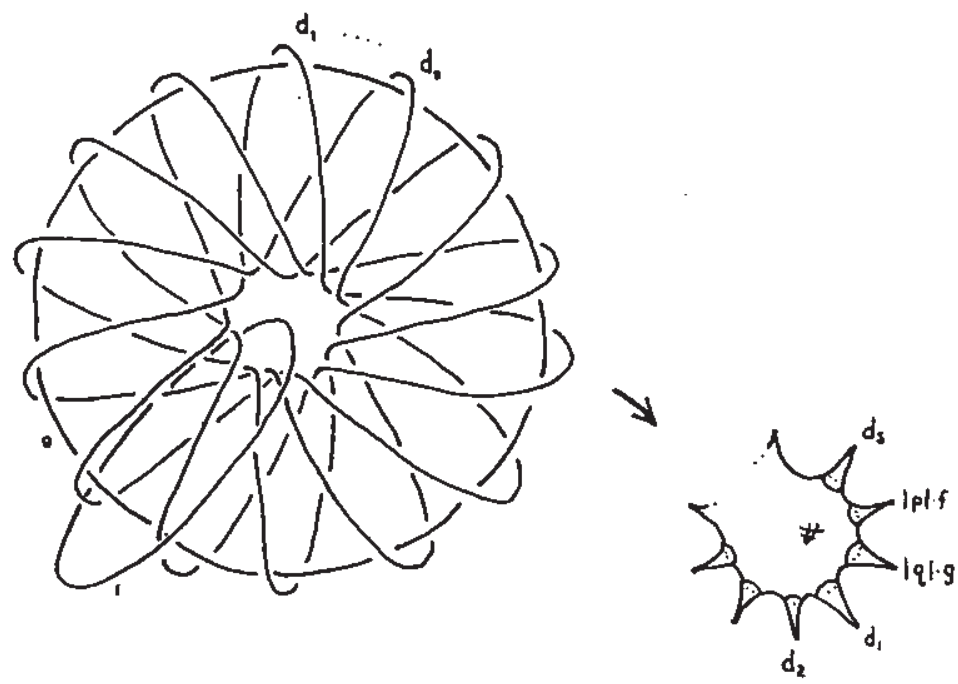

$L\left(p, q: d_{1}, \ldots, d_{s} f, g\right)$

with $p, q \neq 0$, g.c.d. $(p, q)=1, s \geqslant 0, d_{1}>1, \ldots, d_{s}>1, f \geqslant 1, q \geqslant 1$

$(p q>0 \leftrightarrow$ right-handed torus knots; in the figure, $q=2, p=7, s=2$ )

Base 2-orbifold: $S^{2}\left(|p| f,|q| g, d_{1}, \ldots, d_{s}\right)$

Euler characteristic of base $=\bar{\chi}=$

Euler number $=e=-1 / p q(\neq 0)$

$$
2+\sum_{j=1}^{s}\left(-1+1 / d_{i}\right)+(-1+1 /|p| n+(-1+1 /|q| g)
$$

FIGURE 5

$\left.d_{j}, l \leqslant j \leqslant s\right)$, and its Euler characteristic is now

$$
1+(1 / 2) \Sigma_{i=1}^{r}\left(-1+1 / n_{i}\right)+\sum_{j=1}^{s}\left(-1+1 / d_{j}\right) .
$$

The Euler number of the bundle is unaffected by this change.

We will discuss briefly the "obvious" symmetries of these orbifolds (leading to «obvious" repetitions in a naive listing, which can be dealt with in any convenient way). For the moment, our chief concern is that our list be allinclusive; later, geometry by geometry, we will address the question of whether these are "all" the symmetries (the answer is «often yes, sometimes now).

\section{Proposition 3;}

(1) If $\left\{d_{1}, \ldots, d_{s}\right\}=\left\{d_{1}^{\prime}, \ldots, d_{s}^{\prime}\right\}$, then $L\left(p, q: d_{1}, \ldots, d_{s}: f, q\right)=L\left(p, q: d_{1}^{\prime}, \ldots, d_{s}^{\prime} f, g\right)$

(2) $L\left(p, q: d_{1}, \ldots, d_{s}: f, g\right)=L\left(q, p: d_{1}, \ldots, d_{s}: g, f\right)=L\left(-p,-q: d_{1}, \ldots, d_{s}: f, g\right)$

(3) If $q=1$, then $L\left(p, q: d_{1}, \ldots, d_{s} f, g\right)=L\left(p, q: d_{1}, \ldots, d_{s}, g: f, 1\right)$ 
(4) If $p=q=1$, then $L\left(p, q: d_{1}, \ldots, d_{s} f, g\right)=L\left(p, q: d_{1}, \ldots, d_{s} f, g: 1,1\right)$

(5) Reflecting $L\left(p, q: d_{1}, \ldots, d_{s} f, g\right)$ in the plane of projection produces $L\left(p,-q: d_{1}, \ldots, d_{s}: f, g\right)$.
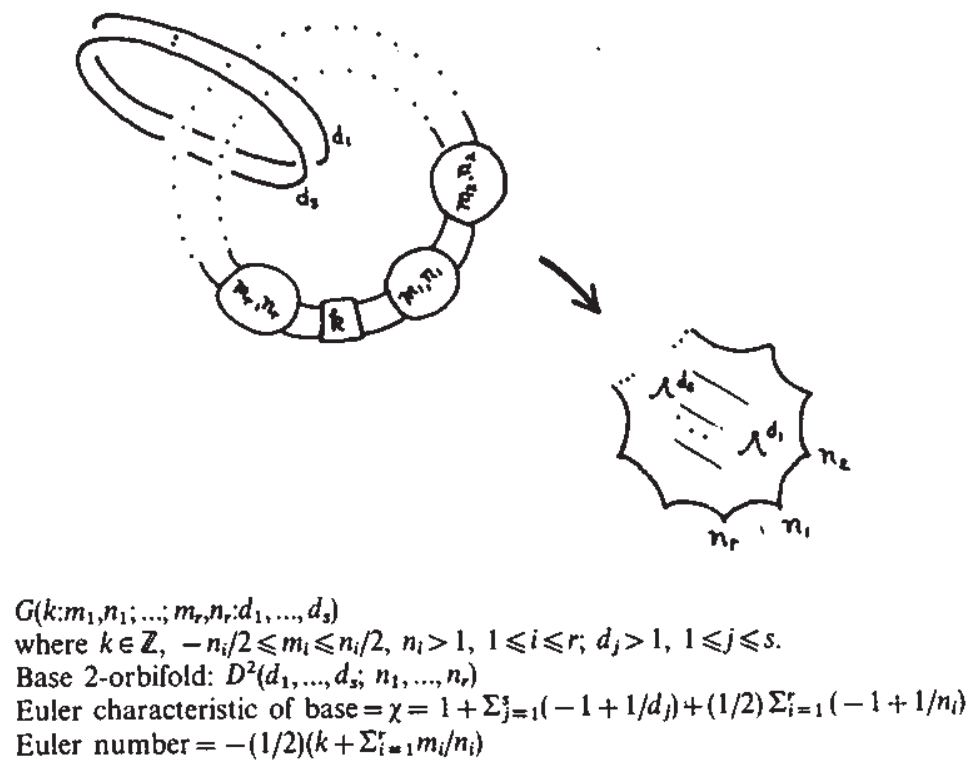

FIGURE 6

\section{Proposition 4:}

(1) If $\left\{d_{1}, \ldots, d_{s}\right\}=\left\{d_{1}^{\prime}, \ldots, d_{s}^{\prime}\right\}$, then

$G\left(k: m_{1}, n_{1} ; \ldots ; m_{r}, n_{r}: d_{1}, \ldots, d_{s}\right)=G\left(k: m_{1}, n_{1} ; \ldots ; m_{r}, n_{r} \cdot d_{1}^{\prime}, \ldots, d_{s}^{\prime}\right)$

(2) $G\left(k: m_{1}, n_{1} ; \ldots ; m_{r}, n_{r}: d_{1}, \ldots, d_{s}\right)=G\left(k: m_{r}, n_{r} ; \ldots ; m_{1}, n_{1}: d_{1}, \ldots, d_{s}\right)$

(3) $G\left(k: m_{1}, n_{1} ; \ldots ; m_{r}, n_{r}: d_{1}, \ldots, d_{s}\right)=G\left(k: m_{2}, n_{2} ; \ldots ; m_{r}, n_{r} ; m_{1}, n_{1}: d_{1}, \ldots, d_{s}\right)$

(4) $G\left(k:-m_{1}, 2 m_{1} ; \ldots ; m_{r}, n_{r}: d_{1}, \ldots, d_{s}\right)=G\left(k-1: m_{1}, 2 m_{1} ; \ldots ; m_{r}, n_{r}: d_{1}, \ldots, d_{s}\right)$

(5) Reflecting $G\left(k: m_{1}, n_{1} ; \ldots, m_{r}, n_{r} \cdot d_{1}, \ldots, d_{s}\right)$ in the plane of projection produces $G\left(-k:-m_{1}, n_{1} ; \ldots ;-m_{r}, n_{r}: d_{1}, \ldots, d_{s}\right)$

Proof: (3) follows from the fact that $|k|$ half-twist can be slid from one side of a tangle to the other, (4) from the fact that a left-handed half-twist can be combined with a tangled graph with parameter $-1,2$ to produce a tangled graph with parameter 1,2 .

\section{Proposition 5:}

(1) $\pi_{1}^{\text {orb }}\left(L\left(p, q: d_{1}, \ldots, d_{s}:, g\right)\right) \simeq<x_{1}, x_{2}, y_{1}, \ldots, y_{s}, h: \quad\left[h, x_{1}\right], \quad\left[h, x_{2}\right], \quad\left[h, y_{j}\right]$, $\left(x_{p}^{p} h^{-b}\right)^{4},\left(x_{2}^{q} h^{-a}\right)^{q}, y^{\left(d_{j}\right)}, x_{1} x_{2} y_{1} \ldots y_{s}>$, where $1 \leqslant j \leqslant s$, and ap $-b q=1$ 
(2) $\pi_{1}^{\text {orb }}\left(G\left(k: m_{1}, n_{1} ; \ldots ; \quad m_{r} n_{r}, d_{1}, \ldots, d_{s}\right)\right) \simeq<x_{1}, \ldots, x_{r}, y_{1}, \ldots, y_{s}, h, c: \quad\left[h, x_{i}\right]$, $\left[h, y_{j}\right], c h c^{-1} h, c^{2},\left(x_{i}^{\left(N_{d}\right)} h^{\left(-M_{i}\right)}\right)^{\left(j_{i}\right)}, y_{1}^{\left(d_{j}\right)}, c x_{i} c^{-1}\left(x_{1} \ldots x_{i-1} x_{i} x_{i-1}^{-1} \ldots x_{1}^{-1}\right)$,

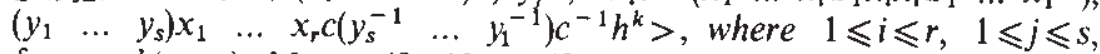
$\delta_{i}=\operatorname{g.c.d} .\left(m_{i}, n_{i}\right), M_{i}=m_{i} / \delta_{i}, N_{i}=n_{i} / \delta_{i}$.

Proof: Part (1) can be viewed as an elaboration on the standard presentations of fundamental groups of Seifert fibered manifolds, using the Seifert-Van Kampen theorem for orbifolds. The expression in part (2) describes $\pi_{1}^{\text {orb }}(G(\ldots))$ as a $\mathbb{Z}_{2}$-extension (via «c») of $\pi_{1}^{\text {orb }}$ of the Seifert fibered orbifold obtained by pulling back along the orientable double cover of the base. It is an elaboration on $[\mathrm{BZ}, 12.33]$.

Remark. The quotient group obtained by adding «h» as a relator in either of the presentations above is $\pi_{1}^{\text {orb }}$ (base 2-orbifold).

\section{TABLE CONVENTIONS}

The diagrams that follow represent singular sets of oriented geometric 3orbifolds whose underlying topological space is $S^{3}$ (with the usual orientation). To reduce clutter, all edges which should be labelled « 2 » have been left unlabelled. If the orbifold has a bona fide "mirror image" (another oriented orbifold such that there is an orientation-reversing diffeomorphism between the two, but no orientation-preserving diffeomorphism), only one of the pair is pictured. If, on the other hand, an orbifold admits an orientationreversing self-diffeomorphism, its picture is labelled with «a», for "amphicheiral». Singular sets which are amphicheiral knots provide an example of this phenomenon.

The tables should have two properties: completeness (all orbifolds having geometric structure $X$ of type $Y$ with underlying space $S^{3}$ appear somewhere in the appropriate table) and nonredundancy (no orbifold appears more than once). To show the former, we can use Theorem 1, plus the remarks at the beginning of $\$ 5$ (for type 2), plus remarks in $\$ 10$ (for type 3), plus ad hoc checking of a finite number of cases (for type 4). The latter property is virtually impossible to achieve in practice, and we settle for lists of «obvious» redundancies (as in Propositions 3 and 4), plus a finite number of special cases. We will be able to show that all repetitions of type 2 and 4 orbifolds, and of the type 3 orbifolds that are $T^{2}$-bundles, have been accounted for. In fact, it seems likely that only obvious redundancies exist in Solv; of. the related results of Sakuma [Sa] on involutions of torus bundles over the circle. Finally, note that proving completeness requires showing that every orbifold labelled as amphicheiral actually is (usually self-evident) and that proving nonredundancy requires showing that every orbifold labelled as nonamphicheiral actually isn't. 


\section{TABLES OF EUCLIDEAN ORBIFOLDS AND NILORBIFOLDS}

These orbifolds will fiber over 2-orbifolds having Euler characteristic zero, which are listed in Table 1 . Note that of the 17 orbifolds, 4 have $S^{2}$ as underlying space (used for type $2 a$ ) and 8 have $D^{2}$ as underlying space (used for type 2b). The type 2 Euclidean orbifolds and nilorbifolds (with underlying space $S^{3}$ ) consist of the (labelled) Seifert links which project to one of the 4 , and of the Montesinos graphs which project to one of the 8. To realize the (non-fibering) Euclidean orbifolds of type 4, we construct fundamental domains for the group actions (as described in [I]), and fold them up. The names of the corresponding crystallographic groups (in International notation, in square brackets) are given for all Euclidean orbifolds (in dimension 2 and 3). Since there are a finite number of affine equivalence classes of crystallographic groups in dimension three (219, of which 54 act preserving orientation), it is a routine matter to show that all the Euclidean 3-orbifolds with underlying space $S^{3}$ have been listed; [Mi, Chapter 2] sketches the proof of the classification theorem.

The second Bieberbach theorem (see [Wo, Thm 3.2.2]) implies that if two Euclidean 3-orbifolds are diffeomorphic (in fact if they have isomorphic fundamental groups), then they are affinely equivalent. Hence, the only Euclidean orbifolds which appear more than once are the ones corresponding to the crystallographic groups $\mathrm{P} 222_{1}$ and C222, each of which appears twice, since each fibers over 2 different bases.

To see that there are no duplications among the nilorbifolds of type $2 \mathrm{a}$ and that the symbol «a» was used correctly, we can use the following facts about their (unlabelled) singular sets (two links in $S^{3}$ are equivalent if there is a homeomorphism of $S^{3}$ which restricts to a homeomorphism of the links).

TABLE 1

Closed 2-orbifolds with $\chi=0$

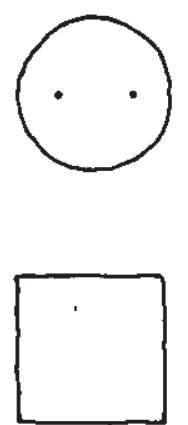

$D^{2}(2,2 ;)$

[pmg]

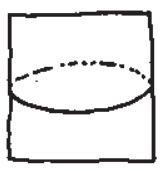

$S^{2}(2,2,2,2)$

$[p 2]$

$D^{2}(; 2,2,2,2)$

[pmm]

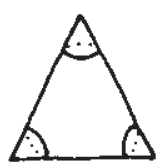


80

William D. Dunbar

TABLE 1. (Continuation)

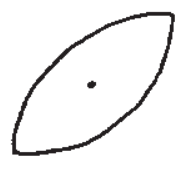

$D^{2}(2 ; 2,2)$
$[c m m]$
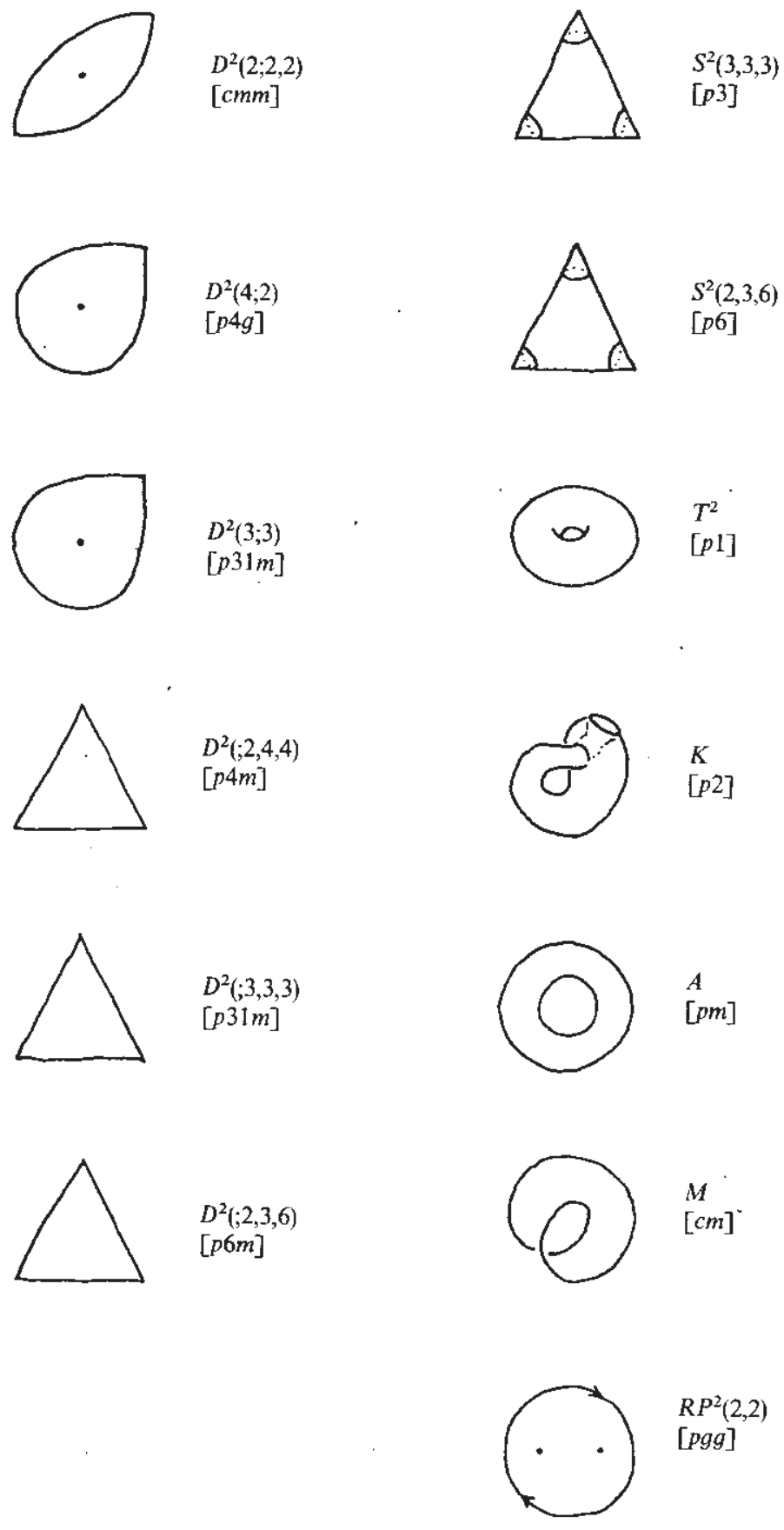
Fibering over $S^{2}(2,2,2,2)$ :
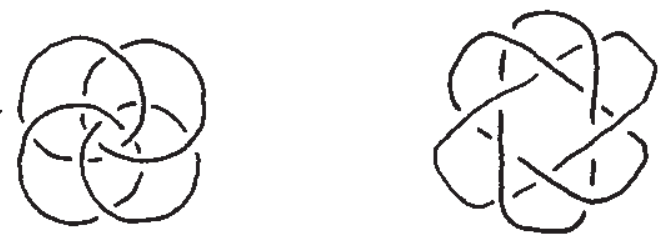

Fibering over $S^{2}(2,4,4)$ :
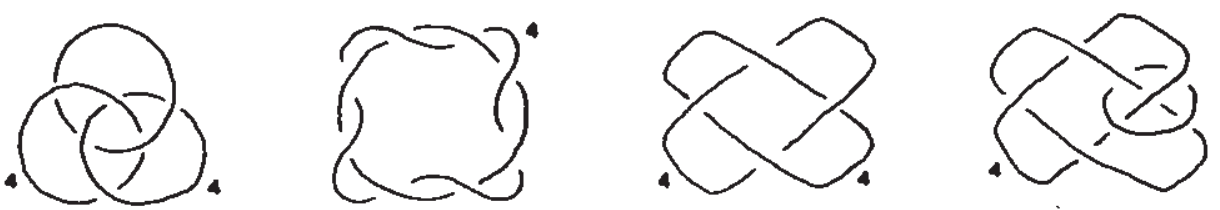

Fibering over $S^{2}(3,3,3)$ :
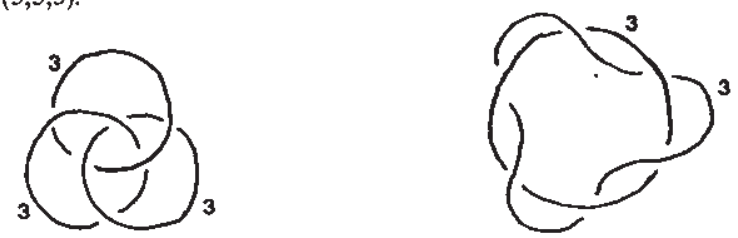

Fibering over $S^{3}(2,3,6)$ :
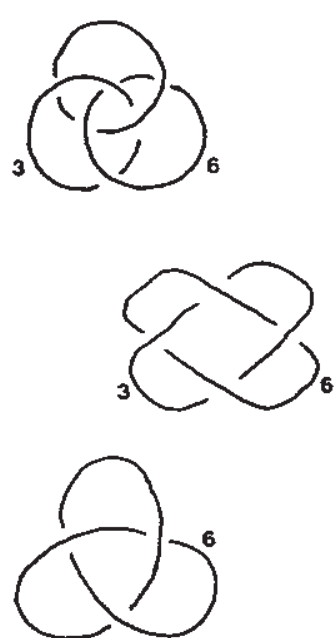<smiles>C1=CCCCCCCCCCC1</smiles><smiles>C1CCCCCCCCCCCCCC1</smiles>

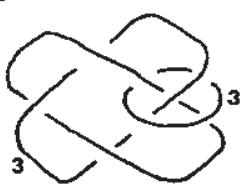

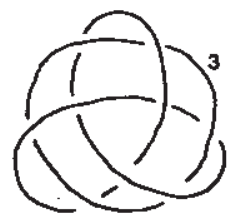


Fibering over $D^{2}(2,2 ;)$ :

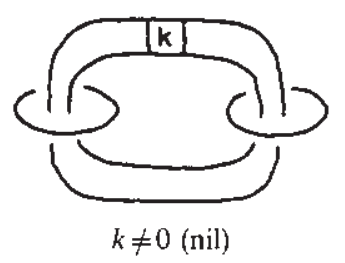

Fibering over $D^{2}(; 2,2,2,2)$ :

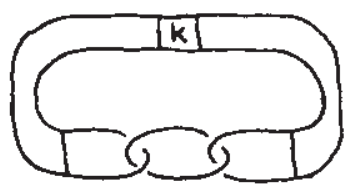

$k \neq-1$ (nil)
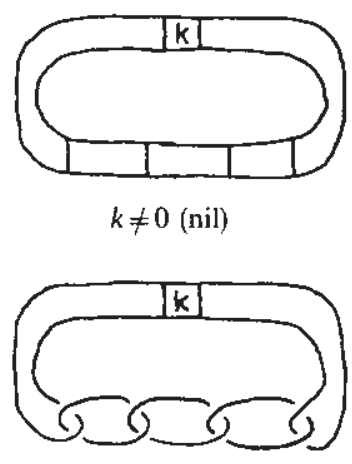

$k \neq-2$ (nil)

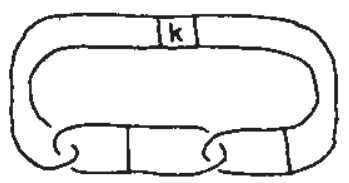

$k \neq-1$ (nil)

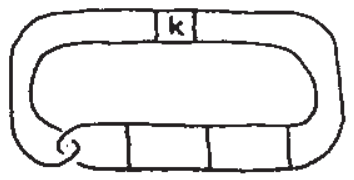

all $k$ (nil)

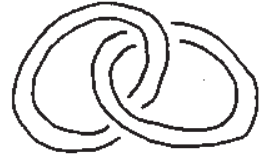

$k=0\left[\mathrm{P} 222_{1}\right]$ 回

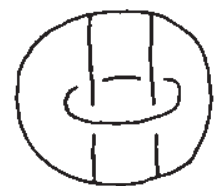

$k=-1[\mathrm{C} 222]$ a

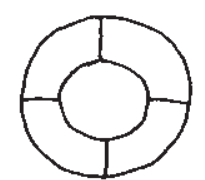

$k=0[\mathrm{P} 222] a$

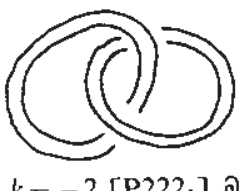

$k=-2[\mathrm{P} 222 \mathrm{~g}]$ 曰

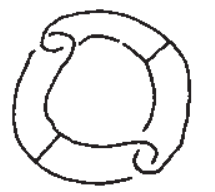

$k=-1[\mathrm{~F} 222]$ a

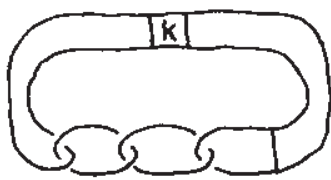

all $k$ (nil) 
TABle 3. (Continuation)

Fibering over $D^{2}(2 ; 2,2)$ :
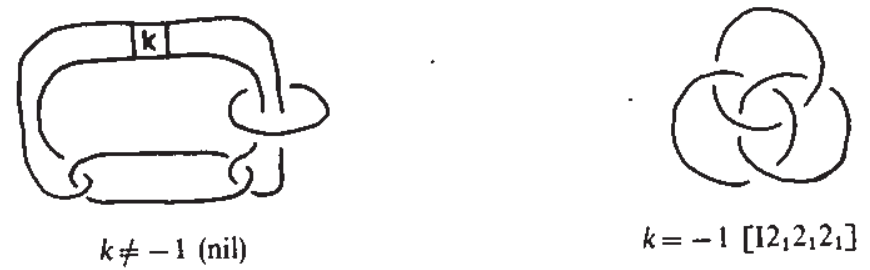

$k=-1[12,2,2$,$] 으$
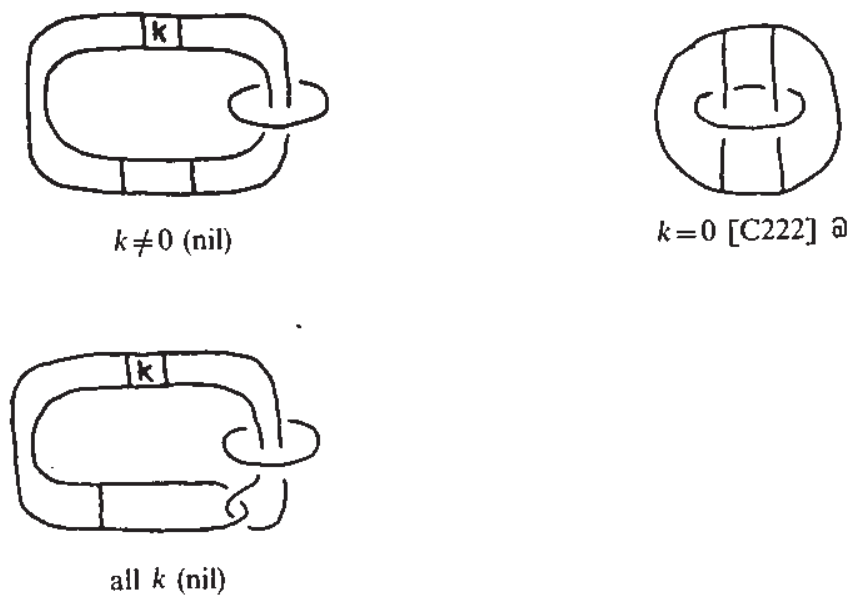

Fibering over $D^{2}(4 ; 2)$ :
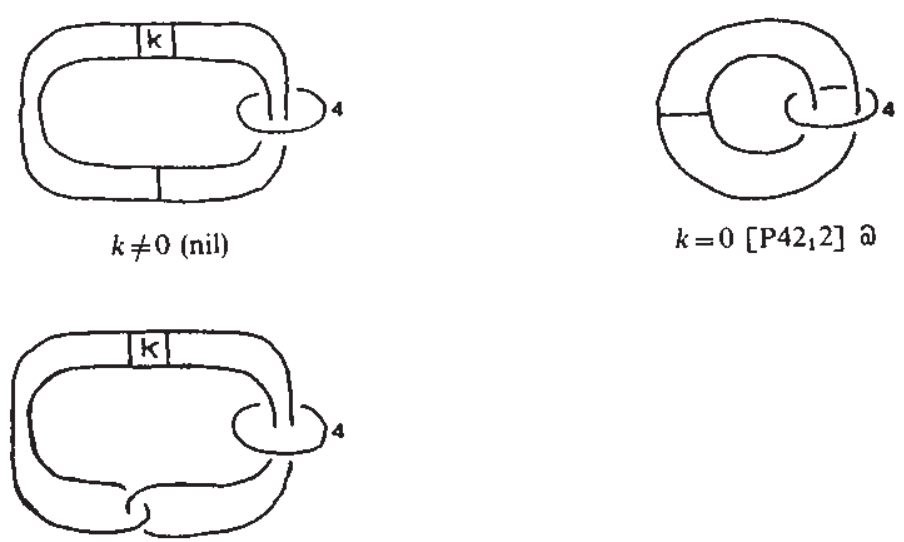

all $k$ (nil) 
TABLE 3. (Continuation)

Fibering over $D^{2}(3 ; 3)$ :
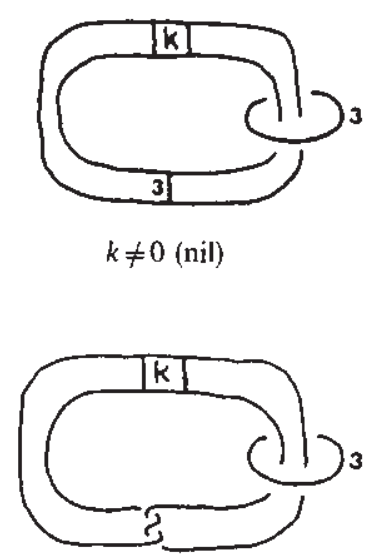

all $k$ (nil)

Fibering over $D^{2}(; 2,4,4)$ :

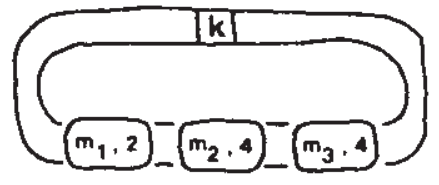

$k+m_{1} / 2+m_{2} / 4+m_{3} / 4 \neq 0$ (nil)

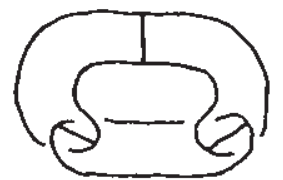

$k=-1, m_{1}=0, m_{2}=m_{3}=2\left[\mathrm{P} 4_{2} 22\right]$ a

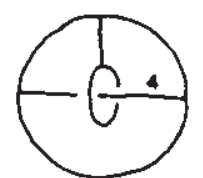

$k=-1, m_{1}=1, m_{2}=0, m_{3}=2[1422]$
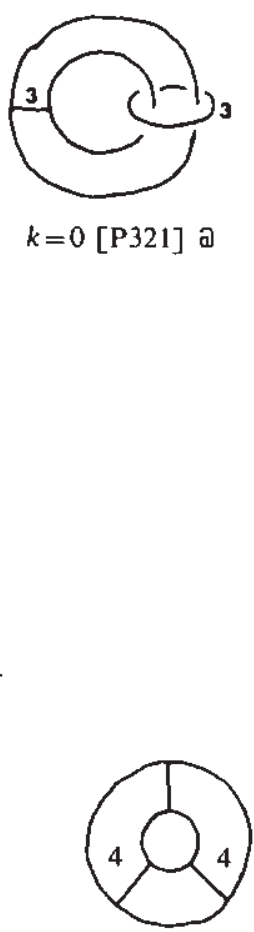

$k=m_{1}=m_{2}=m_{3}=0[\mathrm{P} 422] a$

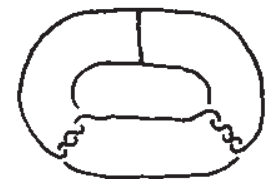

$k=m_{1}=0, m_{2}=1, m_{3}=-1\left[14_{1} 22\right]$ a

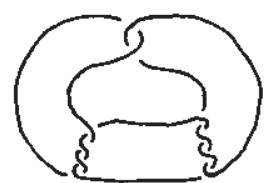

$k=0, m_{1}=1, m_{2}=m_{3}=-1\left[\mathrm{P}_{1} 22\right]$ 
TABle 3. (Continuation)

Fibering over $D^{2}(; 3,3,3)$;

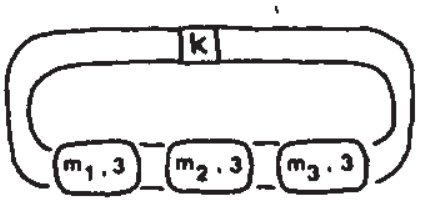

$k+\left(m_{1}+m_{2}+m_{3}\right) / 3 \neq 0$ (nil)

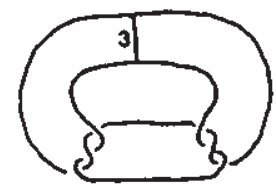

$k=m_{1}=0, m_{2}=-1, m_{3}=1[R 32]$ a

Fibering over $D^{2}(; 2,3,6)$ :

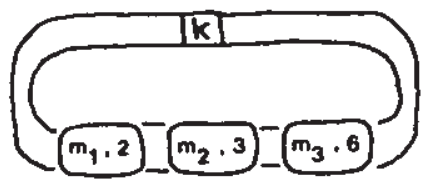

$k+m_{1} / 2+m_{2} / 3+m_{3} / 6 \neq 0($ nil)

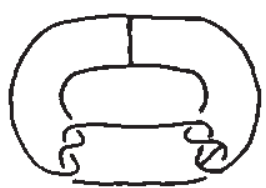

$k=m_{1} 0, m_{2}=1, m_{3}=-2\left[\mathrm{P} 6_{2} 22\right]$

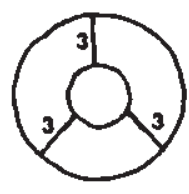

$k=m_{1}=m_{2}=m_{3}=0[\mathrm{P} 312]$ a

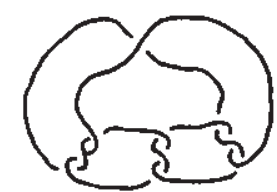

$k=1, m_{1}=m_{2}=m_{3}=-1[P 3,12]$

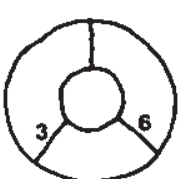

$k=m_{1}=m_{2}=m_{3}=0[\mathrm{P} 622]$ a

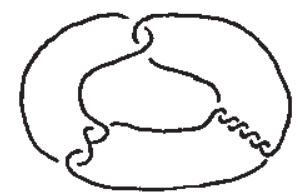

$k=0, m_{1}=1, m_{2}=m_{3}=-1\left[\mathrm{P}_{1} 22\right]$

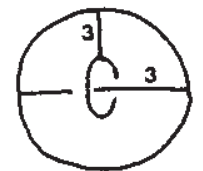

$k=-1, m_{1}=1, m_{2}=0, m_{3}=3\left[\mathrm{P} 6_{3} 22\right]$ a 
TABLE 4

Euclidean orbifolds: type 4

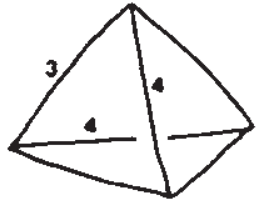

[P432] @

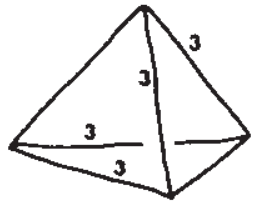

[F23] $\mathrm{a}$

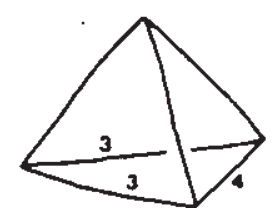

[F432] a

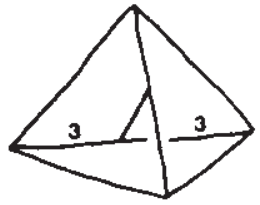

$\left[\mathrm{P}_{2} 32\right]$ क

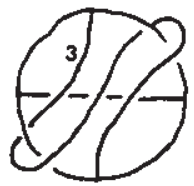

$[14,32]$ a

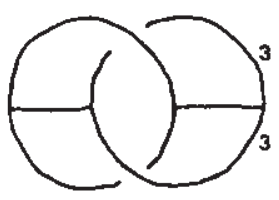

[P23] a

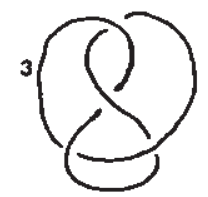

$[P 2,3]$ อ

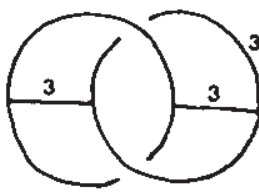

$\left[\mathrm{F}^{4} \mathrm{i} 32\right]$ a

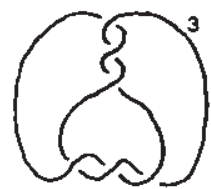

$[12,3]$ a

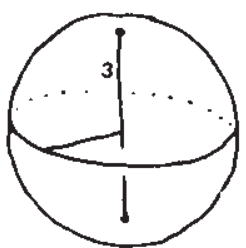

[123] a

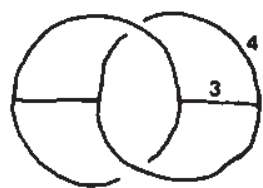

[I432] a

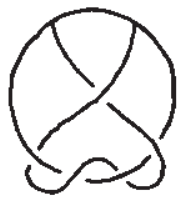

$[\mathrm{P4}, 32]$

(underlying space $=\mathbb{R P}^{3}=3$-ball w/antipodal: $b d y \rightarrow b d y$ ) 
Proposition 6:

(1) A torus knot of type $(p, q)$ is non-trivial iff $|p|$ and $|q|$ are both $>1$.

(2) A non-trivial torus knot is not amphicheiral.

(3) Two non-trivial torus knots of types $(p, q)$ and $\left(p^{\prime}, q^{\prime}\right)$ are equivalent iff $\left(p^{\prime}, q^{\prime}\right)=( \pm p, \pm q)$ or $\left(p^{\prime}, q^{\prime}\right)=( \pm q, \pm p)$.

(4) A link of $s$ components, which are fibers in some fixed Seifert fibering of $S^{3}$, is not amphicheiral if $s \geqslant 3$.

(5) Let $L$ and $L^{\prime}$ be two links, each consisting of $s$ fibers (regular or exceptional) in Seifert fiberings of $S^{3}$ by $(p, q)$ and $\left(p^{\prime}, q^{\prime}\right)$ torus knots respectively (with $0<|q| \leqslant p, 0<\left|q^{\prime}\right| \leqslant p^{\prime}$, g.c.d. $(p, q)=$ g.c.d. $\left.\left(p^{\prime}, q^{\prime}\right)=1\right)$. Let $S:=\#$ of components of $L \cup\left\{\right.$ exceptional fibers\}, let $S^{\prime}$ be similarly defined, and suppose $\min \left\{S, S^{\prime}\right\} \geqslant 3$ ( $S$ and $S^{\prime}$ equal the number of cone points in the base 2-orbifolds, after labelling the components of $L$ and $\left.L^{\prime}\right)$. Then $L$ and $L^{\prime}$ are equivalent iff $p=p^{\prime},|q|=\left|q^{\prime}\right|$ and one of the following holds (in particular, $S=S^{\prime}$ ):

(a) Both links consist entirely of regular fibers.

(b) Both links contain exactly one exceptional fiber, of order $p=p^{\prime}$.

(c) Both links contain exactly one exceptional fiber, of order $|q|=\left|q^{\prime}\right|$.

(d) Both links contain two exceptional fibers.

Proof. Parts (1)-(3) are proven in [BZ, 3.E], and part (4) in [BM]. To prove (5) (the "ifis direction is trivial), assume that $L$ and $L^{\prime}$ are equivalent, and look first at the components of $L$ : you have either $s$ (non-trivial) torus knots of type $(p, q), s-1$ (non-trivial) torus knots of type $(p, q)$ and 1 trivial knot, $s-2$ (non-trivial) torus knots of type $(p, q)$ and 2 trivial knots, or $s$ trivial knots. $S \geqslant 3$ implies that these situations are mutually exclusive. Clearly, $L^{\prime}$ must have the same sorts of components. The first situation falls under case (5a), and using (1)-(3), we can conclude that $p=p^{\prime}$ and $|q|=\left|q^{\prime}\right|$, as desired. . Similarly, the third situation falls under case (5d) and (1)-(3) suffice to complete the argument. In the second situation, we could be either in case (5b) or in case (5c), but these cases can be distinguished by calculating the linking number of the trivial component with one of the others (orient the fibers compatibly); you get $q$ and (sign $q$ ) $\cdot p$, respectively. In the fourth situation, we could be in case (5a) or in case ( 5 b), but linking numbers again serve to resolve ambiguities. Since we want to consider the links as unoriented, we need to note that the magnitudes of the linking numbers suffice to determine the equivalence classes.

Corollary 7. If there is an orientation-preserving diffeomorphism between the two 3-orbifolds $L\left(p, q: d_{1}, \ldots, d_{s}: f, g\right)$ and $L\left(p^{\prime}, q^{\prime}: d_{1}^{\prime}, \ldots, d_{s^{\prime}}^{\prime}: f^{\prime}, g^{\prime}\right)$ with $0<|q| \leqslant p$, $0<\left|q^{\prime}\right| \leqslant p^{\prime}$, g.c.d. $(p, q)=$ g.c.d. $\left(p^{\prime}, q^{\prime}\right)=1, d_{i}>1, d_{\mathrm{i}}^{\prime}>1$ (for $\left.1 \leqslant i \leqslant s, 1 \leqslant i^{\prime} \leqslant s^{\prime}\right)$, $f p>1, g q>1, f p^{\prime}>1, g^{\prime} q^{\prime}>1, \min \left\{S, S^{\prime}\right\} \geqslant 3$, then $p=p^{\prime}, q=q^{\prime}, s=s^{\prime},\left\{d_{1}, \ldots, d_{s}\right\}$ $=\left\{d_{1}^{\prime}, \ldots, d_{s}^{\prime}\right\}, f=f^{\prime}$ and $g=g^{\prime}$. 
[BZ, Thm 12.28] generalizes to show that there are no duplications in the list of nilorbifolds of type $2 b$ (when the Euler number is restricted to positive values); none of these orbifolds is amphicheiral. Finally, there are no nilorbifolds of type $2 a$ which are also of type $2 b$, since the center of $\pi_{1}^{\text {or }}$ is infinite cyclic for the former and trivial for the latter.

\section{SPHERICAL ORBIFOLDS AND $S^{2} \times E^{1}$-ORBIFOLDS}

These orbifolds will fiber over over 2-orbifolds which are quotients of $S^{2}$ by finite subgroups of $O(3)$. The finite subgroups of $O(3)$ are listed (up to conjugacy) in Table 5, using the Schönflies naming convention. Note that of the 14 types (some containing an infinite number of orbifolds, some containing one orbifold), 8 are topologically disks, and 5 are topologically spheres. Several types degenerate to the same orbifold when the parameter $n$ equals 1: $C_{1 v}=C_{1 h}, D_{1}=C_{2}, D_{1 h}=C_{2 v}, D_{1 d}=C_{2 h}$.

TABLe 5

Closed 2-orbifolds with $\chi>0$

$n \geqslant 1$

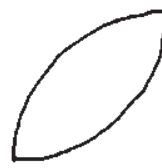

$D^{2}(n, n)$

$\left[C_{m v}\right]$

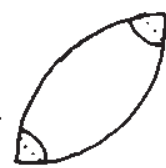

$S^{2}(n, n)$

$\left[C_{n}\right]$

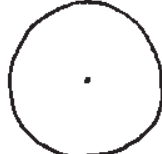

$D^{2}(n ;)$

[C $\left.C_{n h}\right]$

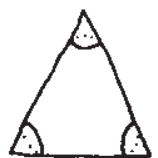

$S^{2}(2,2, n)$

$\left[D_{n}\right]$

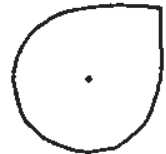

$D^{2}(2 ; n)$

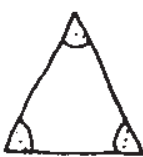

$S^{2}(2,3,3)$

$\left[D_{n d}\right]$

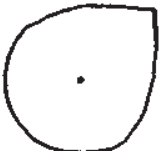

$D^{2}(3 ; 2)$

$\left[T_{d}\right]$

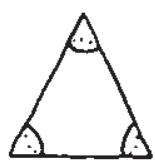

$S^{2}(2,3,4)$

[O]

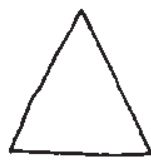

$D^{2}(; 2,2, n)$

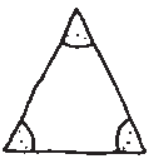

$S^{2}(2,3,5)$

$[I]$ 
TABLE 5. (Continuation)
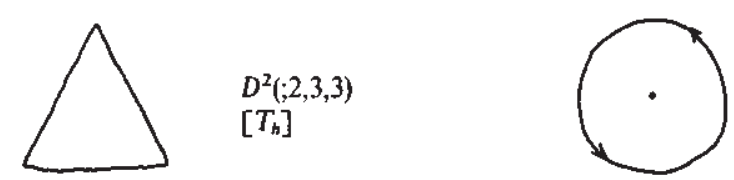

$R P^{2}(n)$

$\left[S_{2 n}\right]$

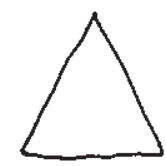

$D^{2}(2,3,4)$

$\left[0_{h}\right]$

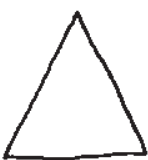

$D^{2}(; 2,3,5)$

$\left[I_{k}\right]$

Bad 2-orbifolds: $S^{2}(m), S^{2}(m, n), D^{2}(; m), D^{2}(; m, n)-$ where $m \neq n .(m, n>1)$

TABLE 6

Spherical orbifolds: type $2 a$

$$
n>1,3 \leqslant a \leqslant 5, k \neq 0
$$

$S^{3}$ (with empty singular set) libers over $S^{2} 0$.

Fibering over $S^{2}(n, n)(f, g \geqslant 1$ are divisors of $n)$ :

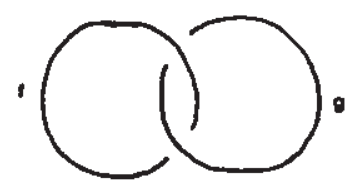

Fibering over $S^{2}(2,2, n)(f \geqslant 1$ is a divisor of $n)$ :

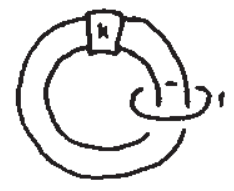


TABLE 6. (Continuation)

Fibering over $S^{2}(2,3, a)$ :
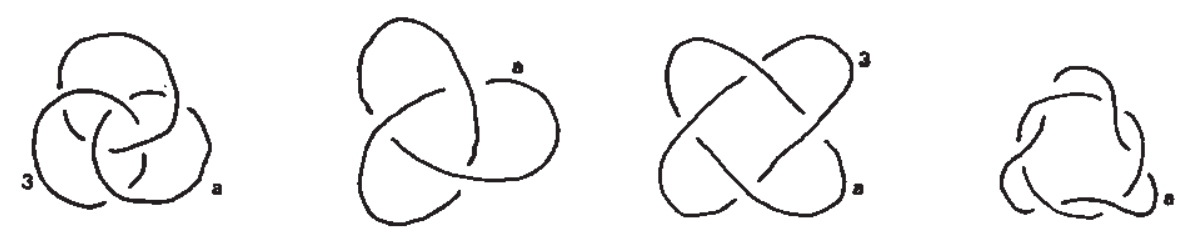

Fibering over $S^{2}(2,3,4)$ :
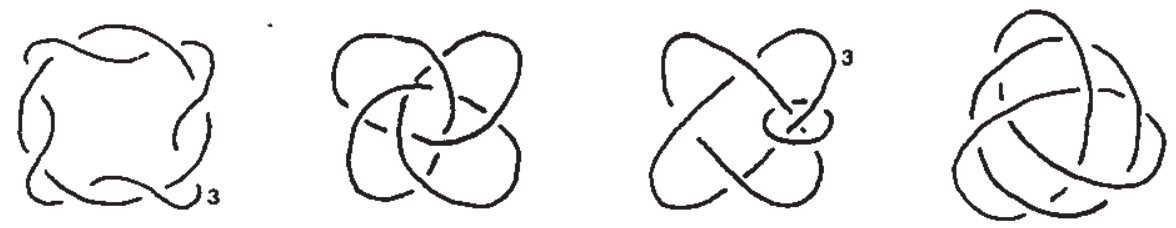

Fibering over $S^{2}(2,3,5)$ :<smiles>c1ccccccccccccc1</smiles>
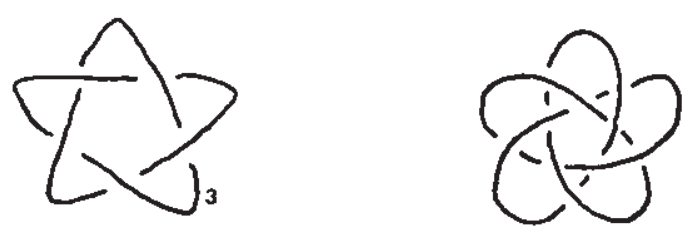

TABLE: 7

. Spherical orbifolds and $S^{2} \times E^{-1}$-orbifolds: $t y p e 2 b$ $n>1$

Fibering over $D^{2}(0)$

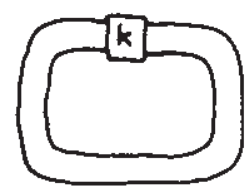

$k \neq 0\left(S^{3}\right)$

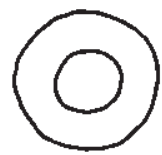

$k=0\left(S^{2} \times E^{1}\right) \square$ 
TABLE 7. (Continuation)

Fibering over $D^{2}(; n, n)$ :

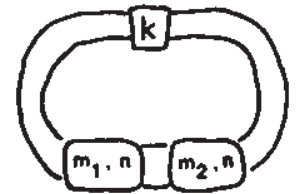

$k+m_{1} / n+m_{2} / n \neq 0\left(S^{3}\right)$

Fjbering over $D^{2}(n ;)$ :

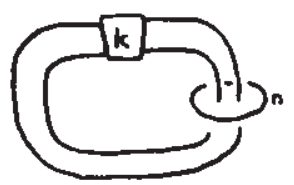

$k \neq 0\left(S^{3}\right)$

Fibering over $D^{2}(2 ; n)$ :

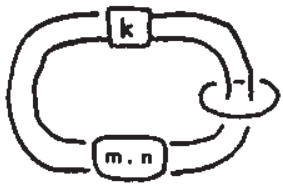

$k+m / n \neq 0\left(S^{3}\right)$

Fibering over $D^{2}(3 ; 2)$;

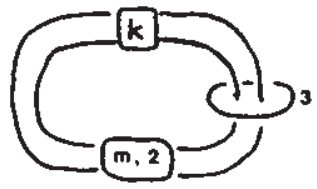

$k+m / 2 \neq 0\left(S^{3}\right)$

Fibering over $D^{2}(; 2,2, n)$

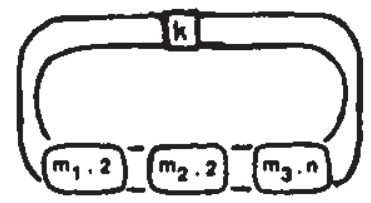

$k+m_{1} / 2+m_{2} / 2+m_{3} / n \neq 0\left(S^{3}\right)$

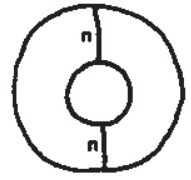

$k=m_{1}=m_{2}=0\left(S^{2} \times E^{1}\right)$ a

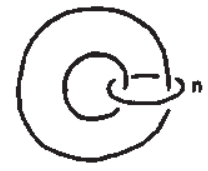

$k=0\left(S^{2} \times E^{1}\right)$

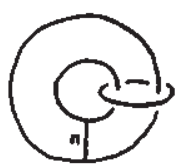

$k=m=0\left(S^{2} \times E_{1}\right)$ a

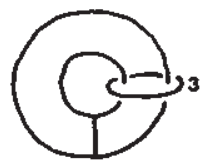

$k=m=0\left(S^{2} \times E^{1}\right)$ a

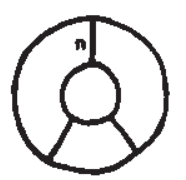

$k=m_{1}=m_{2}=m_{3}=0\left(S^{2} \times E^{1}\right) a$ 
TABLE 7. (Continuation)

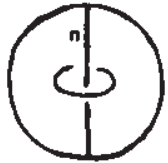

$k=-1, m_{1}=m_{2}=1, m_{3}=0\left(S^{7} \times E^{1}\right)$ D

Fibering over $D^{2}(; 2,3,3)$ :

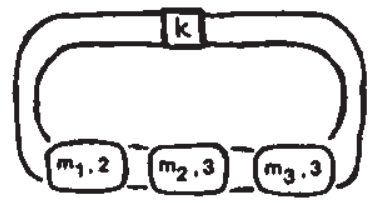

$k+m_{1} / 2+m_{2} / 3+m_{3} / 3 \neq 0\left(S^{3}\right)$

Fibering over $D^{2}(-2,3,4)$ :

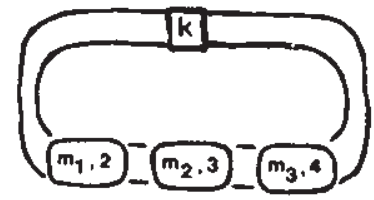

$k+m_{1} / 2+m_{2} / 3+m_{3} / 4 \neq 0\left(S^{3}\right)$

Fibering over $D^{2}(; 2,3,5)$ :

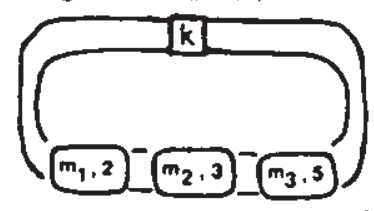

$k+m_{1} / 2+m_{2} / 3+m_{3} / 5 \neq 0\left(S^{3}\right)$

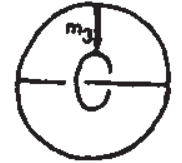

$k=-1, m_{1}=0, m_{2}=1,2 m_{3}=n\left(S^{2} \times E^{1}\right)$ D

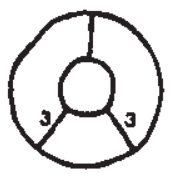

$k=m_{1}=m_{2}=m_{3}=0\left(S^{2} \times E^{1}\right)$ D

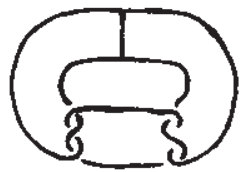

$k=m_{1}=0, m_{2}=1, m_{3}=-1\left(S^{2} \times E^{1}\right)$ a

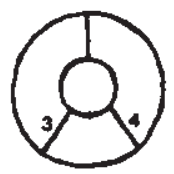

$k=m_{1}=m_{2}=m_{3}=0\left(S^{2} \times E^{1}\right)$ อ

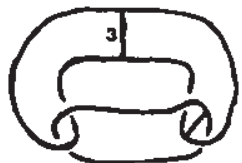

$k=-1, m_{1}=1, m_{2}=0, m_{3}=2\left(S^{2} \times E^{4}\right)$

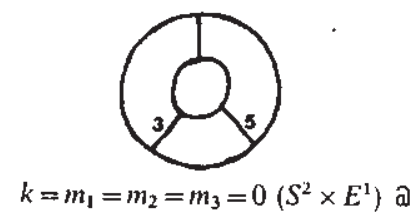




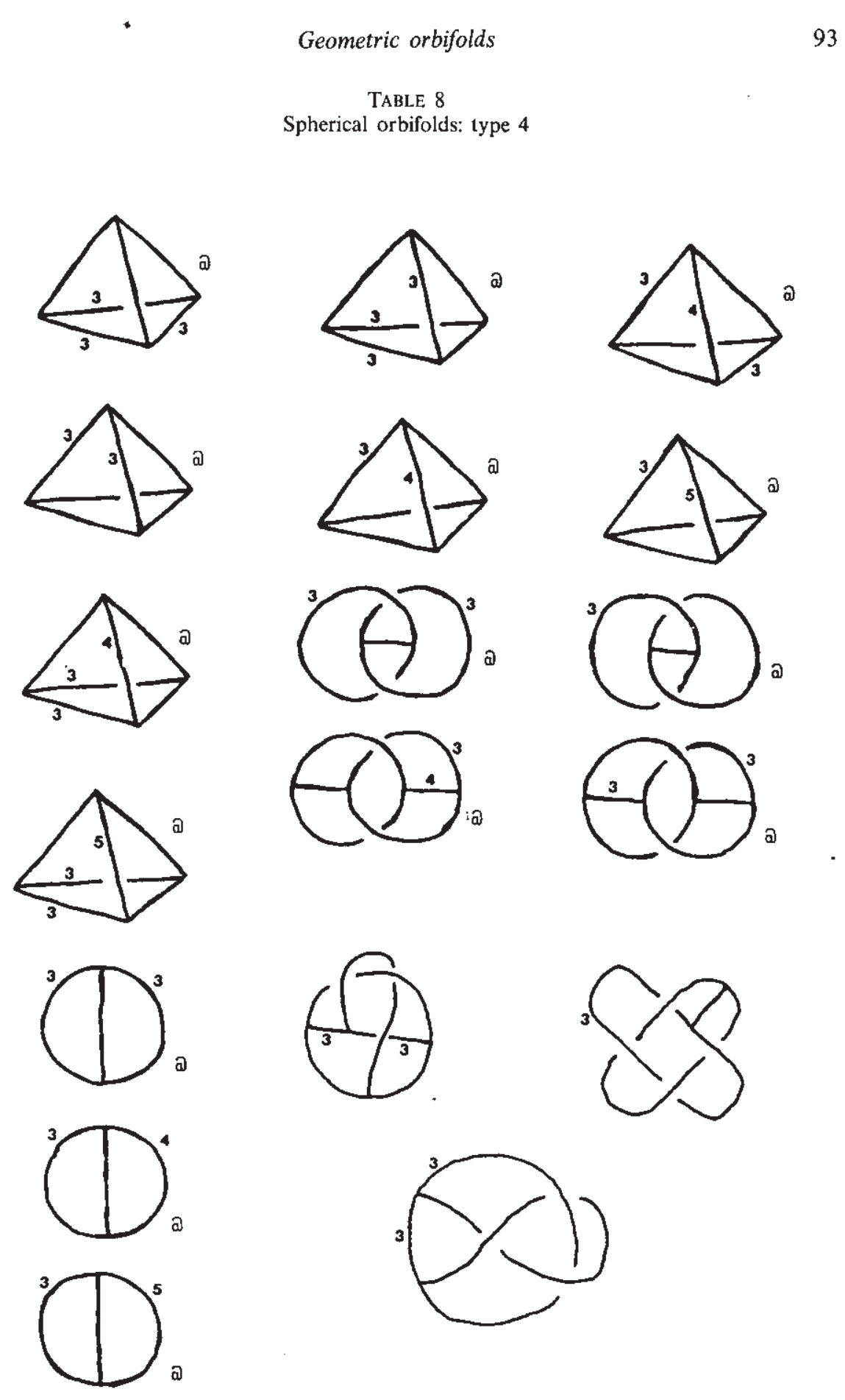


As in the previous section, we use an algebraic classification theorem (in [TS], of the finite subgroups of $\mathrm{SO}(4))$ to determine the groups which will correspond to spherical orbifolds of type 4 , and then fold up fundamental domains for the group actions (on $S^{3}$ ) to find the orbifolds. These groups are characterized by the fact that their images under the 2 maps

$$
\mathrm{SO}(4) \rightarrow \mathrm{SO}(3) \times \mathrm{SO}(3) \stackrel{\mathrm{proj}_{i}}{\longrightarrow} \mathrm{SO}(3), i=1,2,
$$

are both non-cyclic, non-dihedral groups.

One could, without much further trouble, name the «spherical crystallographic groups" (using the convention of [TS]) corresponding to the spherical orbifolds in these tables. We hope to do this in a future article.

The listed $S^{2} \times E^{1}$-orbifolds can mostly be distinguished from each other by comparing singular sets on combinatorial grounds (edge labels, number of vertices, number of components). Upon inspection, only one duplication is uncovered, that of the orbifold whose singular set consists of a theta-graph and a circle, with all edges labelled « $2 »$, arising from the «obvious» symmetry $G(-1: 0,2 ; 1,2 ; 1,2 ;)=G(-1 ; 1,2 ; 1,2 ; 0,2 ;)$.

The spherical orbifolds are trickier to sort out. A rigidity theorem due to DeRham (see [Ro, Thm 4.3]) says that diffeomorphic spherical orbifolds of any dimension are in fact isometric. Hence the only problem is that of finding the same kind of degeneracies that occurred when enumerating the spherical 2-orbifolds (i.e., different descriptions of the same subgroup of $\mathrm{SO}(4)$ ). Here are a few duplications that crept in:

$$
\begin{aligned}
& L(4,3: 2: 1,1)=G(-1: 1,2 ; 1,3 ; 1,3:) \\
& L(5,3: 2 ; 1,1)=G(-1: 1,2 ; 1,3 ; 1,5:) \\
& L(3,2: 2: 1,2)=G(-1: 1,2 ; 1,3 ; 1,4:) \\
& G(k-1: 1,2 ; 1,2 ; m, n:)=\left\{\begin{array}{lll}
G(0:-n, k n+m: 2) & \text { if } & k+(m / n)>0 \\
G(0 ; n,-k n-m: 2) & \text { if } & k+(m / n)<0
\end{array}\right. \\
& G(k: n)=\left\{\begin{array}{lll}
L(-k / 2,1: 2,2: n, 1) & k \text { even } \\
L(-k, 2: 2: n, 1) & k \text { odd }
\end{array}\right.
\end{aligned}
$$

Also, there are many ways to represent the same 2-bridge link (possibly with struts connecting the pairs of bridges at each end) as a Montesinos graph $G\left(k: m_{1}, n ; m_{2}, n:\right)$.

\section{9. $H^{2} \times E^{1}$-ORBIFOLDS AND $T_{1}\left(H^{2}\right)$-ORBIFOLDS}

In terms of displaying the orbifolds, not much improvement can be made over the figures in $\$ 5$, since hyperbolic base orbifolds are «generic». The facts used in $\S 7$ to weed out duplications and to prove non-amphicheirality apply to this case as well. 


\section{SOLVORBIFOLDS}

As mentionned in Theorem 1, solvorbifolds all fiber over either the circle or $\mathrm{A}:=$ circle/reflection, with generic fiber either $T^{2}$ or $S^{2}(2,2,2,2)$. Thus, any solvorbifold is either the mapping torus of some self-map of $T^{2}$ (resp. $S^{2}(2,2,2,2)$ ), or can be split into 2 orbifolds-with-boundary which are of the form $\left(T^{2} \times I\right) /$ involution reversing both factors) (resp. $\left.\left(S^{2}(2,2,2,2) \times I\right) /(\ldots)\right)$ by removing a regular neighborhood of a (generic) fiber. There are only a finite number of these pieces, up to diffeomorphism. Reversing the process yields all solvorbifolds (plus some nilorbifolds and some Euclidean orbifolds). Consequently, it is clear that only those fibering over $\square$ can have underlying space $S^{3}$, most coming from gluing together two twisted I-bundles over $D^{2}(; 2,2,2,2)$, $D^{2}(2,2 ;)$, or $D^{2}(2 ; 2,2)$ (each having the 3-ball as underlying space), and a few coming from gluing together two twisted I-bundles over the annulus or the Möbius band - with boundary points modelled on $\mathbb{R}^{2} /$ reflection - (each having a solid torus as underlying space).

In the former case, all gluing maps can be considered as elements of the mapping class group of the four-times-punctured sphere, which is a (split) extension of $\mathbb{Z}_{2} \times \mathbb{Z}_{2}$ by $\operatorname{PSL}(2, \mathbb{Z})$. Because $\operatorname{PSL}(2, \mathbb{Z})$ is isomorphic to $\mathbb{Z}_{2} * \mathbb{Z}_{3}$, we can find nice representatives of conjugacy classes, of the form

$$
(\alpha) \beta^{ \pm 1} \alpha \beta^{ \pm 1} \alpha \ldots \beta^{ \pm 1} \gamma \text {, where } \gamma \in \mathbb{Z}_{2} \times \mathbb{Z}_{2} \text {. }
$$

The final refinement comes from noting which elements of the mapping class group (acting on the boundary) extend across each of the I-bundles. The idea is to incorporate the $\alpha$ on the left end of the word and the $\gamma$ on the right end into the "standard» picture for the I-bundles. The braid $\alpha$ can be pushed into the twisted I-bundle over $D^{2}(2,2 ;)$ only at the cost of allowing two alternative ways to draw it, since $\alpha$ does not extend across this bundle (it does extend over the other two bundles). Similarly, two elements of $\mathbb{Z}_{2} \times \mathbb{Z}_{2}$ do not extend across the I-bundle over $D^{2}(2 ; 2,2)$, and we need two alternative pictures for this bundle. Thus there are 25 a priori subcases $(5=1+2+2$ choices for the top, 5 for the bottom). Making use of the symmetries to be described in Proposition 9 and the following remark, we can reduce consideration to 11 subcases listed in Figure 7.

\begin{tabular}{l|ccc} 
bottom & $D^{2}(2,2,2,2)$ & $D^{2}(2,2 ;)$ & $D^{2}(2 ; 2,2)$ \\
\hline$D^{2}(2,2,2,2)$ & 1 & 2,3 & 7 \\
$D^{2}(2,2 ;)$ & & $4,5,6$ & 8,9 \\
$D^{2}(2 ; 2,2)$ & & & 10,11
\end{tabular}

FIGURE 7 
These words (read left to right) in the letters $\alpha, \beta$, and $\beta^{-1}$, can be translated into 3 -strand braids (read top to bottom) in the «letters» given in Figure 8 .

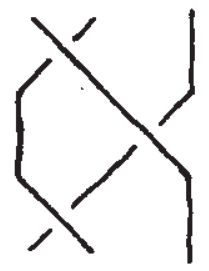

$\beta$

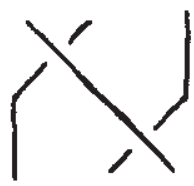

FIGURE 8 $\beta^{-1}$

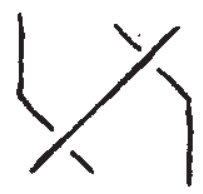

In fact, with the reductions now made, the word can be described by giving the successive exponents of $\beta$. There are exceptional sequences yielding Euclidean and nilorbifolds, which fall into three classes:

subcases 1-6:

$$
+1,+1, \ldots,+1 \text { or }-1,-1, \ldots,-1 \text { (of length } \geqslant 1 \text { ) or } \varnothing
$$

subcases 7-9:

$$
\begin{aligned}
& +1,+1, \ldots,+1,-1 \text { or }-1,-1, \ldots,-1,+1 \text { (of length } \geqslant 2 \text { ) or }+1 \text { or }-1 \\
& \text { or } \varnothing
\end{aligned}
$$

subcases 10-11:

$$
-1,+1,+1, \ldots,+1,-1 \text { or }+1,-1,-1, \ldots,-1,+1 \text { (of length } \geqslant 3 \text { ) or }
$$$$
+1,+1 \text { or }-1,-1 \text { or }+1 \text { or }-1 \text { or } \varnothing
$$

The following proposition notes an obvious collection of symmetries (giving a sufficient condition for amphicheirality).

Proposition 8: In subcases 1,4 and 10 , reflecting a solvorbifold (with $\beta$ exponent sequence $\mathscr{S}$ ) in the $x z$-plane (taking the plane of the paper to be the $x y$-plane) produces a solvorbifold in the same subcase with $\beta$-exponent sequence $-\mathscr{P}$, read backwards.

Proof. Immediate using $\alpha=\alpha^{-1}$.

Proposition 9: In subcases 1,5,6, and 10, rotating a solvorbifold (with $\beta$ exponent sequence $\mathscr{S}$ ) by $180^{\circ}$ about a horizontal line in the plane of the paper produces a solvorbifold in the same subcase with $\beta$-exponent sequence $\mathscr{P}$ read backwards.

Proof. Immediate, since the «letters» $\alpha$ and $\beta$ are mapped to $\alpha$ and $\alpha \beta \alpha$ respectively. 

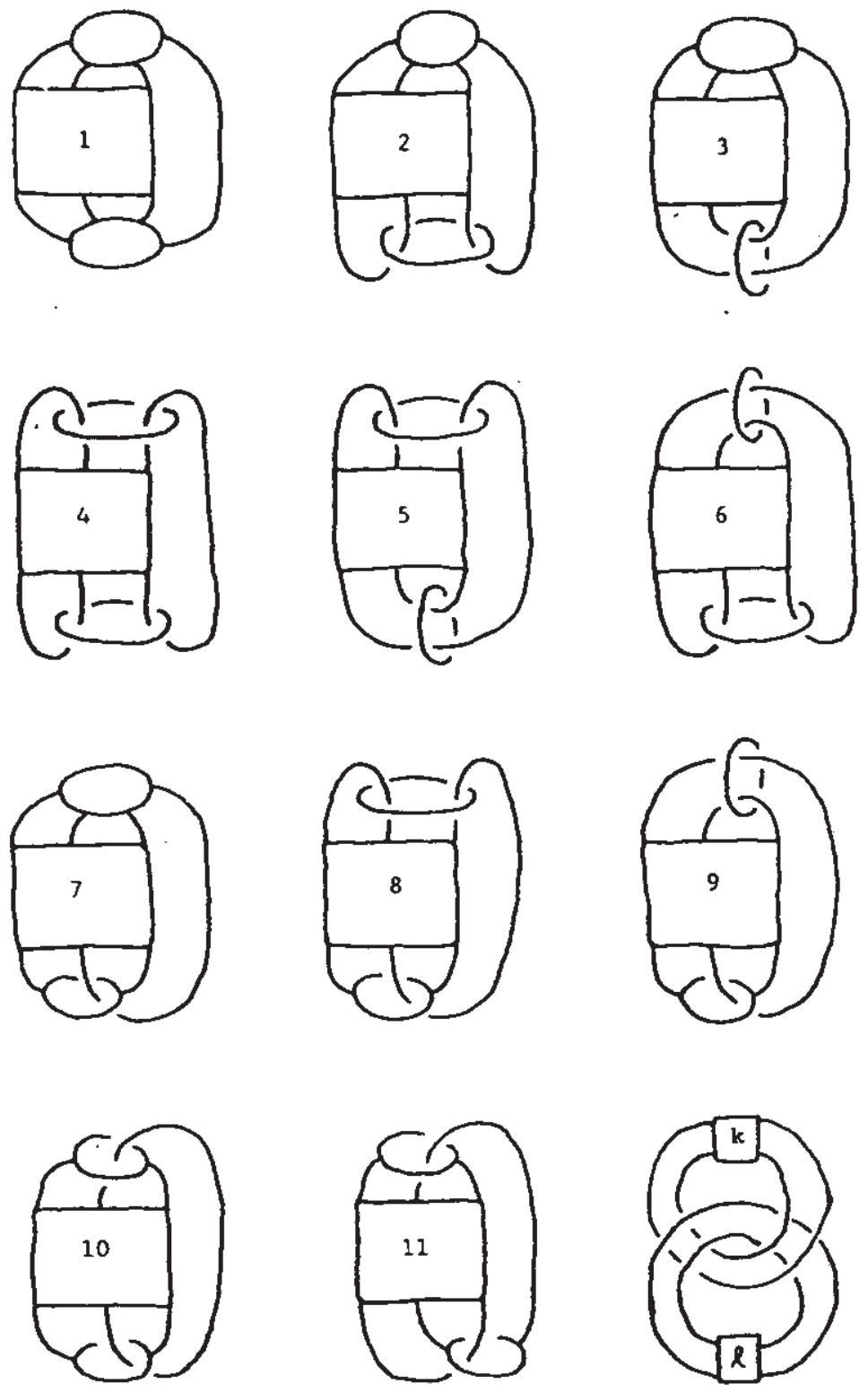

$|k|-2 \mid>2$ 
Remark. Another way to alter an orbifold in Table 9 so that it appears different is to add $\gamma \gamma$, for some $\gamma \in \mathbb{Z}_{2} \times \mathbb{Z}_{2}$, at the beginning or end of the word, and then use conjugacy relations to push one of the $\gamma^{\prime}$ s to the other end of the word, at which point it has become a possibly different element $\gamma^{\prime} \in \mathbb{Z}_{2} \times \mathbb{Z}_{2}$. This is most useful when one of the ends is a twisted I-bundle over $D^{2}(2 ; 2,2)$.

The $T^{2}$-bundles over $\rrbracket$ are all distinct (except for the obvious orientation-preserving symmetry interchanging $k$ and $l$, and the obvious orientation-reversing symmetry negating both $k$ and $l$ ), as one can verify using the classification of 2-bridge knots and linking numbers of the components with each other (well-defined up to sign, since the components of the links are unoriented). Calculating of the monodromy of the $T^{2}$-bundles over $S^{1}$ which double-cover distinguishes any remaining cases (e.g., $k=1, l=5$ from $k=-1$, $l=5$ ). In particular, such an orbifold is an amphicheiral solvorbifold iff $k=-l$ (and $k \neq 0$ ).

\section{REFERENCES}

[BM] Burde, G. and Murasugi, K., Links and Seifert fiber spaces, Duke Math. J., 37 (1970), 89-93.

[BS1] BONAHON, F. and SIEBENMANN, L., Geometric splittings of classical knots, and the algebraic knots of Conway, L.M.S. lecture note series, to appear.

[BS2] BONAHON, F. and SIEBENMANN, L., Seifert 3-orbifolds and their role as natural crystalline parts of arbitrary compact irreducible 3-orbifolds, preprint (1983).

[BS3] Seifert fibered 3-orbifolds, in Low dimensional Topology, ed. Roger Fenn, LMS Lecture Note Series \#95, Cambridge Univ, Press (1985).

[BZ] Burde, G. and ZIeschang, H., Knots, de Gruyter Studies in Mathematics \# 5, Walter de Gruyter, Berlin (1985).

[Co] CONWAY, J. H., An enumeration of knots and links, and some of their algebraic properties, in Computational Problems in Abstract Algebra (ed. J. Leach), Pergamon Press (1970).

[DM] DAvis, M. and MORGAN, J., Finite group actions on homotopy 3-spheres, in The Smith Conjecture (ed. J. Morgan and H. Bass), Academic Press (1984).

[Du] Dunbar, W., Fibered orbifolds and crystallographic groups, thesis, Princeton Univ. (1981).

[HLM]HILDEN, H., LOZANo, M. T., MONTESinos, J., The Whitehead link, the Borromean rings, and the knot $9_{46}$ are universal, Collect. Math., 34 (1983), 1928.

[I] International tables for $x$-ray crystallography (ed. K. Lonsdale), volume I, Kynoch Press, Birmingham, England (1967).

[Mi] MILlER, W., Symmetry groups and their applications, Academic Press (1972).

[Mo] Montesinos, J., Revêtements ramifiés de noeuds, espaces fibrés de Seifert et scindements de Heegaard, preprint, Orsay (1978).

[MM] MCCullough, D. and MiLler, A., Manifold coters of 3-orbifolds wirh yoometric pieces, preprint (1986). 
[Oe] OeRtel, U., Closed incompressible surfaces in complements of star links, Pac. J. Math., 111 (1984), 209-230.

[Ro] ROTHENBERG, M., Torsion invariants and finite transformation groups, 267-311, in Proceedings of Symposia in Pure Mathematics, volume XXXII, part 1, AMS (1978).

[Sa] SAKUMA, M., Involutions on torus bundles over $S^{1}$, Osaka J. Math., 22 (1985), 163-185.

[Sc] SCOTT, G. P., The geometries of 3-manifolds, Bull. London Math. Soc., 15 (1983), 401-487.

[Se] SEIFERT, H., Topologie dreidimensionaler gefaserter Räume, Acta Math., 60 (1933), 147-238.

[Th1] THURSTON, W, Three-dimensional manifolds, Kleinian groups, and hyperbolic geometry, Bull. Amer. Math. Soc., 6 (1982), 357-382.

[Th2] ThuRston, W., Three-dimensional geometry and topology, mimeographed notes, Princeton Univ. (1978).

[Th3] THURSTON, W., Hyperbolic structures on 3-manifolds, I, II...

[Th4] THuRston, W., Three-manifolds with symmetry, preprint (1982).

[TS] THRELFALL, W, and SEIFERT, H., Topologische Untersuchung der diskontinuitätsbereiche endlicher Bewegungsgruppen des dreidimensionalen sphärischen Raumes, I and II, Mathematische Annalen 104 and 107 (1930 and 1932), 1-70 and 543-586.

[Wo] Wolf, J, Spaces of constant curvature, 4th edition, Publish or Perish (1977).

Department of Mathematics

University of Michigan

Ann Arbor, Michigan 48109-1003. USA 